\title{
Robo recruitment of the Wave Regulatory Complex plays an essential and conserved role in midline repulsion
}

Karina Chaudhari ${ }^{1}$, Madhavi Gorla ${ }^{1}$, Chao Chang $^{2,3}$, Artur Kania $^{2.3}$ and Greg J. Bashaw ${ }^{1 *}$

${ }^{1}$ Department of Neuroscience, Perelman School of Medicine, University of Pennsylvania, Philadelphia, PA 19104, USA.

${ }^{2}$ Institut de recherches cliniques de Montréal (IRCM), Montréal, QC, H2W 1R7, Canada.

${ }^{3}$ Department of Anatomy and Cell Biology and Division of Experimental Medicine, McGill University, Montréal, QC, H3A 2B2, Canada.

${ }^{*}$ Corresponding author and Lead Contact:

Greg J. Bashaw

Tel: 215-898-0829

E-mail: gbashaw@pennmedicine.upenn.edu

Running Title: The WRC is required for midline repulsion

Keywords: Axon Guidance; Spinal Cord; Midline; Repulsion; Roundabout; Robo; Slit; Wave Regulatory Complex; Arp2/3; WIRS motif; Receptor Signaling; Growth Cone 


\section{SUMMARY}

The Roundabout (Robo) guidance receptor family induces axon repulsion in response to its ligand Slit by inducing local cytoskeletal changes; however, the link to the cytoskeleton and the nature of these cytoskeletal changes are unclear. Here we show that the heteropentameric Scar/Wave Regulatory Complex (WRC) which drives Arp2/3-induced branched actin polymerization, is a direct effector of Robo signaling. Biochemical evidence shows that Slit triggers WRC recruitment to the Robo receptor's WIRS motif. In Drosophila embryos, mutants of the WRC enhance Robo1-dependent midline crossing defects. Additionally, mutating Robo1's WIRS motif significantly reduces receptor activity in rescue assays in vivo, and CRISPR-Cas9 mutagenesis shows that the WIRS motif is essential for endogenous Robo1 function. Finally, axon guidance assays in mouse dorsal spinal commissural axons demonstrate that the WIRS motif is also required for Robo1 repulsion in mammals. Together, our data support an essential conserved role for the WRC in commissural axon repulsion. 


\section{INTRODUCTION}

The brain is the most complex organ in the body, with trillions of synapses specified by the precise targeting of axons and dendrites during nervous system development. Axons are guided to their appropriate targets by a number of conserved guidance cues and their receptors, which enable neurons to form specific connections to establish functional neural circuits. The axon guidance receptors that mediate axonal guidance and targeting are tightly regulated to achieve a controlled balance between attractive and repulsive signaling and failure to do so results in a number of movement disorders and other neurological deficits (Bosley et al., 2005; Depienne et al., 2011; Jen et al., 2004). Specifically, the Roundabout (Robo) family of axon guidance receptors has been implicated in many neurodevelopmental disorders like Autism Spectrum Disorder, Dyslexia, Horizontal Gaze Palsy and others (Anitha et al., 2008; Hannula-Jouppi et al., 2005; Jen et al., 2004; Suda et al., 2011). Elucidating the mechanisms by which these guidance receptors function is crucial for understanding the formation of neural circuits both during development and in disease pathogenesis.

The Drosophila midline is analogous to the vertebrate spinal cord and serves as an intermediate target for commissural axons that cross from one side of the body to the other (Klambt et al., 1991; Seeger et al., 1993). The Drosophila ventral nerve cord has a ladder-like structure consisting of 13 repeated segments, each containing an anterior commissure and a posterior commissure into which commissural neurons extend their axons to cross the midline. Midline glial cells secrete a number of guidance cues that act on their cognate receptors present on axon growth cones to induce attraction toward or repulsion away from the midline. Slit is secreted by midline glia and acts as a repulsive ligand for the Robo family of receptors (Battye et al., 1999; Brose et al., 1999; Kidd et al., 1999; Kidd et al., 1998). There are three Robo receptors in Drosophila and four in vertebrates. The Robo receptors are transmembrane proteins with an ectodomain consisting of five immunoglobulin-like domains and three 
fibronectin repeats, and an intracellular domain containing short, highly conserved cytoplasmic (CC) motifs (Bashaw et al., 2000; Kidd et al., 1998). Robo1 induces repulsion in growth cones of navigating axons primarily by modulating the actin cytoskeletal network. Previous work has identified some downstream effectors for Robo1 including Ena, an uncapping protein for actin filaments (Bashaw et al., 2000), and Son of Sevenless (SOS), a GEF for Rac1 (Yang and Bashaw, 2006). However, downstream signaling of Robo1 is not completely understood, especially in relation to effectors that directly link Robo1 to the actin cytoskeleton and the nature of cytoskeletal changes orchestrated by Robo1. While it seems intuitive for repulsive signaling to induce depolymerization of the actin network, a recent study reports that dorsal root ganglion axons first extend actin-rich filopodia toward a source of Slit before retracting away from it (McConnell et al., 2016). This challenges the prevailing notion that repulsive signaling primarily relies on actin depolymerization and suggests that the actin rearrangements occurring downstream of Robo1 are more nuanced and complex than previously thought. Indeed, several of the well-known downstream effectors of Robo1 signaling, namely Ena and Rac1, are documented enhancers of actin polymerization (Barzik et al., 2005; Ridley et al., 1992).

The Scar or WAVE regulatory complex (WRC) is a heteropentameric complex consisting of five different proteins: Scar/WAVE, Cyfip/Sra1, Kette/Nap1, HSPC300/Brick1 and Abi (Eden et al., 2002). Scar or WAVE contains a VCA (verprolin homology, cofilin homology, acidic) region and serves as a nucleation-promoting factor for Arp2/3 thereby driving branched actin polymerization. While mammals have multiple orthologs of these proteins, Drosophila has single homologs of all five members of the complex, making it a simpler, more tractable model system for studying the WRC. The WRC has been previously implicated in axon guidance and targeting in Drosophila and C. elegans (Shakir et al., 2008; Stephan et al., 2011; Xu and Quinn, 2012), however if and how it is recruited and activated downstream of guidance receptors is not known. Recent work identified a unique binding site for the WRC known as WRC-interacting receptor sequence (WIRS) motif (Chen et al., 2014a). The WIRS motif is a short six amino-acid 
peptide sequence characterized by a threonine or a serine followed by a phenylalanine. The WIRS motif is present in a number of transmembrane proteins including Robo1 (Chen et al., 2014a). Robo1 has a WIRS motif between its CCO and CC1 domains that is conserved across species, including humans. Previously, the WIRS motif has been shown to be important for the recruitment of the WRC by Neuroligins and SYG-1 in synapse formation (Chia et al., 2014; Xing et al., 2018) and for Neogenin function in maintaining the stability of adherens junctions (Lee et al., 2016). To our knowledge, this study is the first to demonstrate a role for the WIRS motif in recruiting the WRC during axon guidance.

Here, we show that the WRC is required for Slit-Robo1 repulsive signaling at the Drosophila midline. We present evidence that Robo1 interacts with the WRC partially via its WIRS motif and that this interaction is enhanced in the presence of Slit. We show that the WIRS motif in Robo1 is important for its ability to induce ectopic repulsion in vivo. Using rescue assays, we show that Robo1 also requires its WIRS motif to restore repulsion in ipsilateral axons in vivo. In addition, using CRISPR-Cas9-mediated mutagenesis, we show that the WIRS motif is important for endogenous Robo1 function as mutating the endogenous WIRS motif results in loss of Robo1 repulsion at the midline. Finally, we use mouse dorsal spinal cord explants and growth cone collapse assays in mouse commissural neurons to demonstrate that the WIRS motif is also important for vertebrate Robo1 repulsive signaling. We propose a model in which Slit binding induces recruitment of the WRC to the WIRS motif of Robo1 where it functions in Robo1-mediated repulsion at the midline.

\section{RESULTS}

The WRC interacts genetically with slit, robo1 and sos

WRC members are enriched in the Drosophila ventral nerve cord during embryonic stages 12 to 17 , encompassing the developmental window when midline crossing decisions are being made (Schenck et al., 2004). To confirm these previously published observations, we examined the 
expression of Scar by immunofluorescence and observed strong axonal staining throughout embryonic stages when midline axon guidance occurs (Figure S1A). To investigate the potential role of the WRC in Slit-Robo repulsion at the midline, we tested for genetic interactions between cyfip and hspc300, two members of the WRC, and the Slit-Robo signaling pathway. In wild type embryos, Fasll-positive ipsilateral axons project longitudinally and never cross the midline (Figure 1A). In robo1 mutants, axons in the medial most Fas-II bundle frequently cross and recross the midline resulting in a very strong ectopic crossing phenotype (Kidd et al., 1998). In slit, robo1/+ embryos, where the slit and robo1 gene dosage is reduced by half, the phenotype is milder (Figure 1C). This represents a sensitized background in which we can detect enhancers and suppressors of the Slit-Robo pathway (Chance and Bashaw, 2015; Coleman et al., 2010; Fan et al., 2003; Hsouna et al., 2003). While we see no crossing errors in Fasll-positive axons in $h s p c 300$ mutants alone (Figure 1B), in the slit, robo1/+sensitized background, $h s p c 300$ mutants exhibit a significant enhancement of the ectopic crossing defects (Figure 1D). These interactions are dosage sensitive as removing one copy of $h s p c 300$ results in a moderate enhancement of crossing errors while removing both copies of $h s p c 300$ results in a much stronger phenotype (Figure 1I). Similarly, we see almost no crossing errors in Fasll-positive axons in cyfip mutants alone (Figure 1E) however, in the slit, robo1/+sensitized background, cyfip mutants show a strong dose-dependent enhancement of the ectopic crossing defects (Figure 1F and 1G). Strikingly, removing both copies of cyfip in this background results in a very strong phenotype with ectopic crossing defects in nearly $100 \%$ of segments, similar to the robo 1 mutant phenotype (Figure $1 \mathrm{G}$ and $1 \mathrm{~J}$ ). These ectopic crossing defects can be significantly rescued by the transgenic expression of UAS-CYFIP using the pan-neuronal elav-Gal4 driver (Figure $1 \mathrm{H}$ ). This suggests that the neuronal function of CYFIP is important for Slit-Robomediated repulsion at the midline. It is important to note that zygotic $h s p c 300$ and cyfip mutants, like mutants for all other members of the WRC, still have significant amounts of the protein remaining due to maternal deposition (Schenck et al., 2004; Zallen et al., 2002). This likely 
explains why these zygotic mutants have no phenotype on their own._This can be seen in scar zygotic mutants where the overall Scar protein level is significantly reduced but there is still a considerable amount of Scar protein remaining in central nervous system (CNS) axons (Figure S1B and S1C).
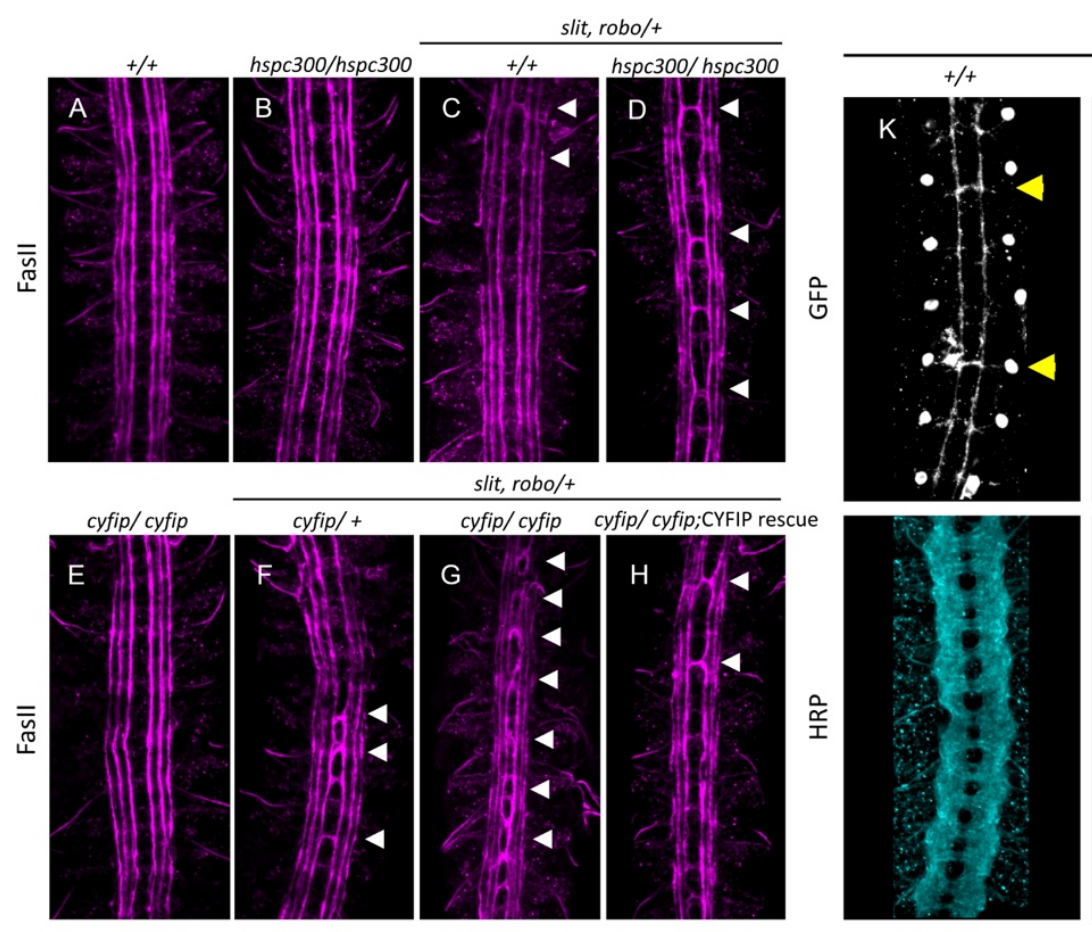

slit, robo, apCD8GFP/+
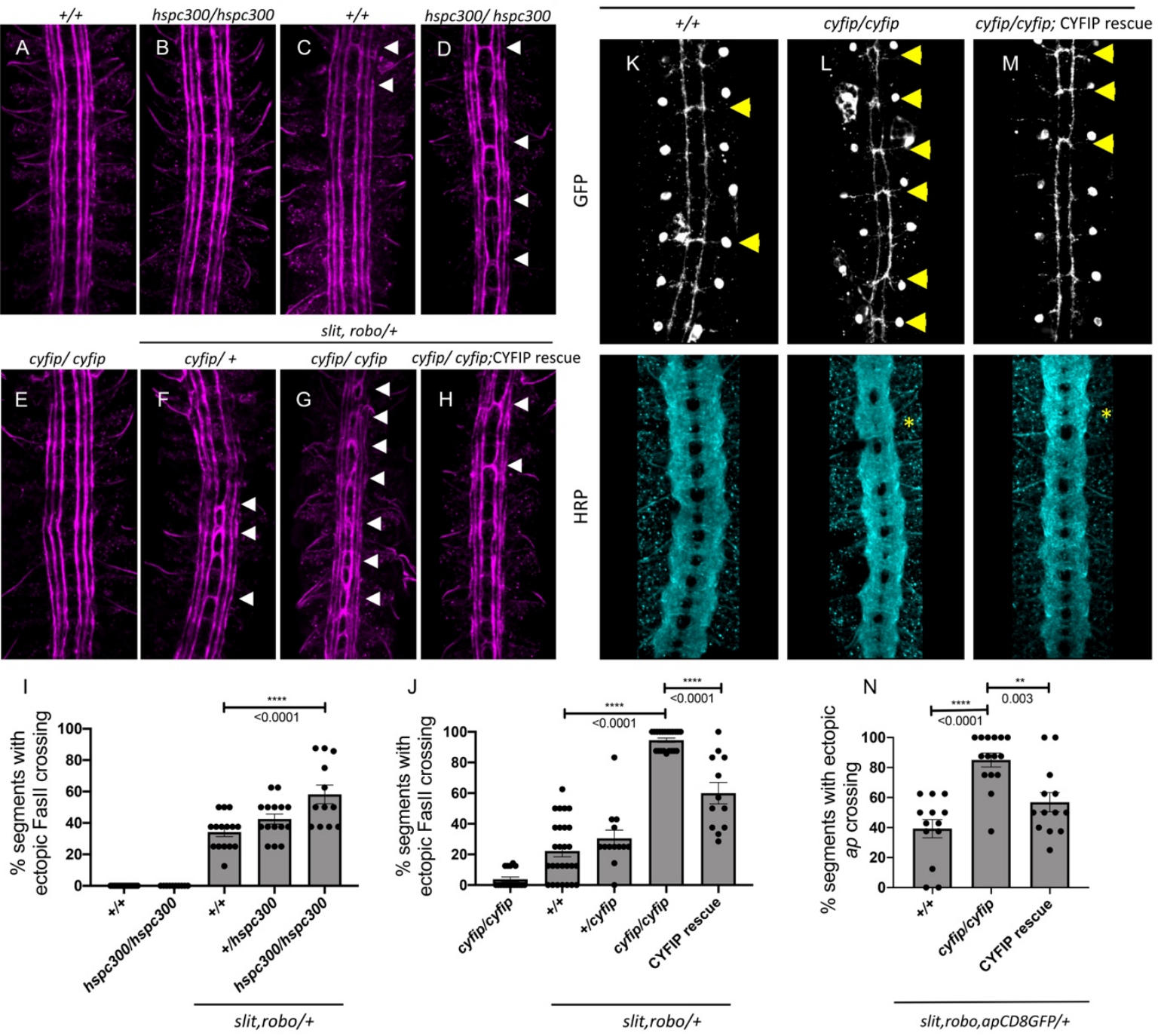

Figure 1. The wave regulatory complex genetically interacts with slit and robo. (A-H) Stage 17

Drosophila embryos stained with anti-Fasll to label ipsilateral axons. (A and B) Wild type and $h s p c 300$ homozygous mutant embryos show three Fasll-positive tracts that do not cross the midline. (C) Double heterozygous slit, robo embryos show a mild loss-of-repulsion phenotype with ectopic Fasll crossing in $34 \%$ of nerve cord segments (arrowheads). (D) hscp300 embryos heterozygous for slit and robo have more segments of the nerve cord with ectopic Fasll crossing. (E) cyfip embryos have almost no ectopic crossing defects and appear like wild type embryos. Removing (F) one and (G) two copies of cyfip in a slit, robo background results in a dose dependent enhancement of the ectopic Fasll crossing defects. (H) Driving UAS-CYFIP expression in neurons using the pan-neuronal elav-Gal4 driver results in a partial rescue of the ectopic Fasll crossing defects. (I and J) Quantitation shows the percentage of segments in 
which Fasll axons ectopically cross the midline. Data are presented as mean $\pm \mathrm{SEM}$, number of embryos, $\mathrm{n}=15,10,15,15,12$ (for I) and 17, 27, 13, 21, 12 (for J). Significance was assessed using ANOVA with Tukey's multiple comparisons test. (K-M) Stage 17 embryos stained with anti-GFP which labels the apterous (ap) cell bodies and axons that normally project ipsilaterally and anti-HRP which labels all CNS axons. (K) Double heterozygous slit, robo embryos show a mild ectopic ap crossing phenotype (yellow arrowheads) while HRP depicts a wild type arrangement of longitudinal and commissural axon pathways. (L) cyfip homozygous mutants in a slit, robo background show a strong enhancement of the ectopic ap crossing defects and HRP shows abnormal thickening and fusion of the commissures (asterisk). (M) Apterous-specific expression of UAS-CYFIP significantly rescues the ectopic ap crossing defects but not the pan-neuronal HRP defects. (N) Quantitation shows percentage of segments with ectopic apterous crossing defects. Data are presented as mean $\pm S E M$, number of embryos, $n=13,15$, 13. Significance was assessed using one-way ANOVA with Tukey's multiple comparisons test.

To determine whether CYFIP is required cell-autonomously, we examined a more restricted subset of ipsilateral axons, the apterous (ap) axons. Just like the Fasll axons, ap axons are sensitive to a partial loss of repulsion. Reducing the slit and robo1 gene dosage by half in slit, robo1/+ embryos results in a mild phenotype where ectopic midline crossing of ap axons is seen in approximately $40 \%$ of segments (Figure $1 \mathrm{~K}$ ). Homozygous cyfip mutants in this sensitized background show a strong enhancement of the ectopic ap crossing defects with $85 \%$ of segments exhibiting ectopic crossing (Figure 1L). We also visualized all CNS axons using HRP and observed abnormal thickening and fusion of the commissures, a phenotype which bears strong resemblance to robo1 mutants. Importantly, Ap-specific expression of UAS-CYFIP significantly rescues the ectopic ap crossing defects but not the pan-neuronal HRP defects (Figure $1 \mathrm{M}$ and $1 \mathrm{~N}$ ) providing strong support for a cell autonomous role for CYFIP in Slit-Robo1 signaling. These genetic data suggest that the WRC functions in the Slit-Robo1 pathway.

Next we predicted that the simultaneous reduction of CYFIP and SOS, another signaling effector of Robo1, would greatly impair Robo1-mediated repulsion, resulting in axons ectopically crossing the midline. SOS is a well-established downstream effector of Robo1 and is required for its axon guidance function at the midline (Chance and Bashaw, 2015; Yang and Bashaw, 2006). As SOS is also maternally deposited (Yang and Bashaw, 2006), zygotic sos mutants show very mild ectopic crossing defects in approximately $15 \%$ of segments (Figure $2 \mathrm{~A}$ ). In contrast, double mutants for sos and cyfip show a striking phenotype in which Fasll-positive 
axons ectopically cross the midline in over $80 \%$ of segments (Figure 2B and $2 \mathrm{E}$ ), a phenotype which bears strong resemblance to the robo1 mutant phenotype. In addition to examining the phenotype with Fasll immunostaining, we also visualized all CNS axons using HRP and observed frequent thickening and fusion of the anterior and posterior commissures, which again bears strong resemblance to robo1 mutants (Figure 2B). Thus, cyfip genetically interacts with sos to give a strong ectopic crossing phenotype very similar to that seen in robo1 mutants.

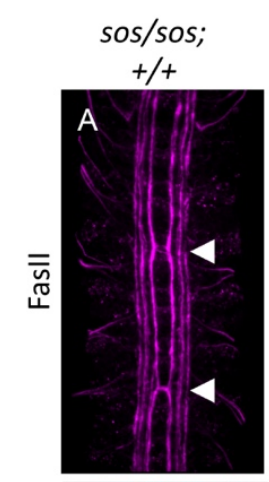

sos/sos;
cyfip/cyfip
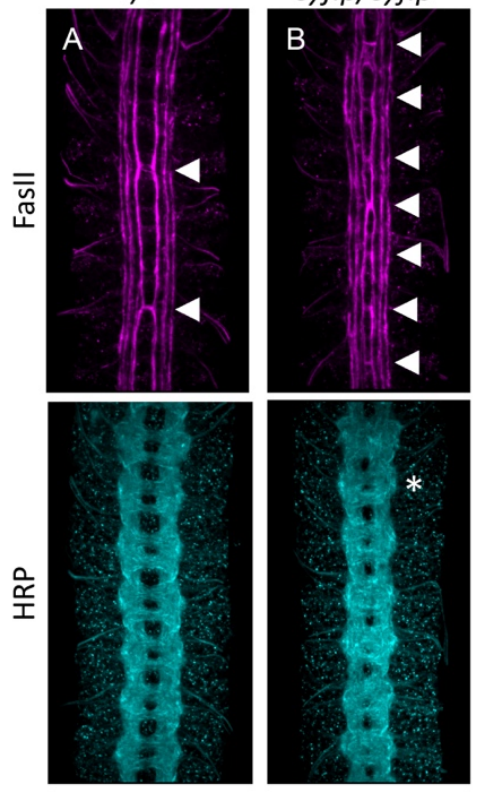

$\mathrm{E}$

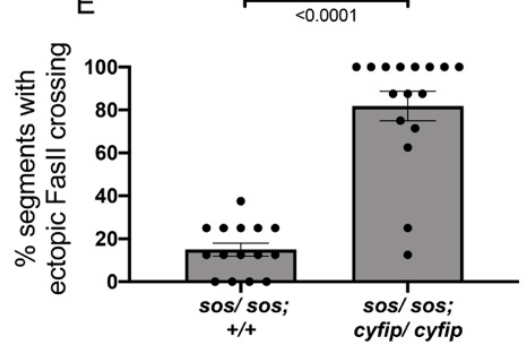

robo2/robo2;

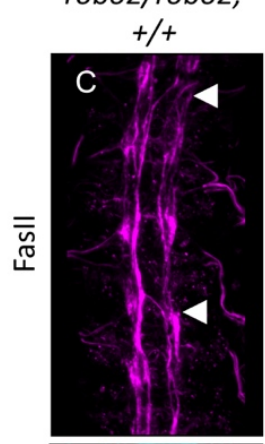

robo2/robo2;

cyfip/cyfip
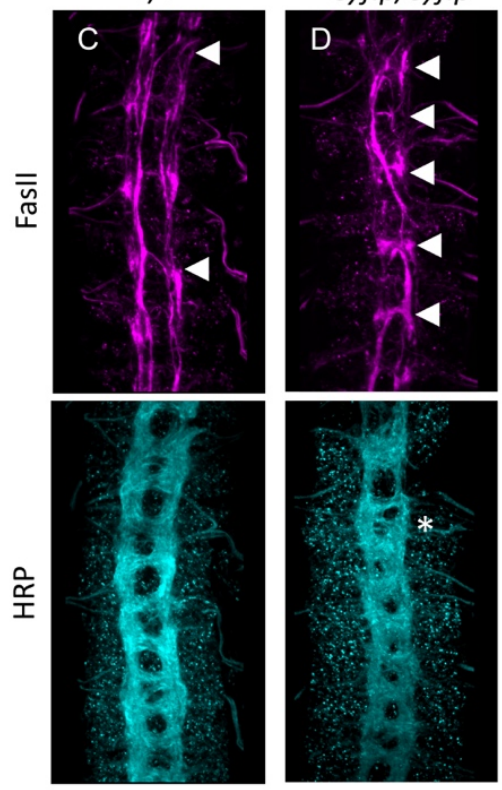

$\mathrm{F}$

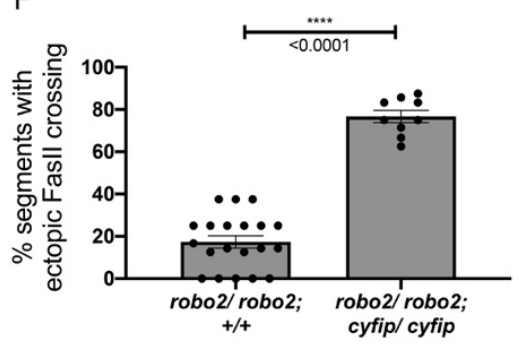

Figure 2. The wave regulatory complex genetically interacts with sos and robo2. (A-D) Stage 17 embryos stained with anti-Fasll and anti-HRP. (A) sos embryos show mild ectopic crossing defects in Fasll axons (arrowheads) and no phenotype in HRP. (B) Simultaneous removal of sos and cyfip results in a very strong enhancement of the ectopic Fasll crossing defects and a strong HRP phenotype with thickening and fusion of commissures (asterisk). Similarly, (C) robo2 mutants show mild ectopic crossing defects in Fasll axons and a mildly disorganized axon scaffold in HRP while (D) double mutants for robo2 
and cyfip show strong ectopic Fasll crossing defects and thickening and fusion of commissures. (E and F) Quantitation shows the percentage of segments in which Fasll axons ectopically cross the midline. Data are presented as mean \pm SEM, number of embryos, $n=15$ and 16 (for $E$ ) and 20 and 9 (for $F$ ). Significance was assessed using Student's $t$-test.

In Drosophila embryos, both Robo1 and, to a lesser extent, Robo2 contribute to midline repulsion in response to Slit (Rajagopalan et al., 2000; Simpson et al., 2000). Indeed, on their own robo2 mutants exhibit only mild phenotypes; however, robo1, robo2 double mutants exhibit a complete collapse of all CNS axons at the midline, phenocopying the slit mutant phenotype. Therefore, mutations in genes that contribute to robo1 repulsion would be expected to strongly enhance the mild phenotype observed in robo2 mutants. In robo2 mutant embryos, Fasllpositive axons ectopically cross the midline in approximately $17 \%$ of segments (Figure $2 \mathrm{C}$ ). In robo2, cyfip double mutant embryos, ectopic crossing defects are greatly increased to approximately $75 \%$ of segments (Figure $2 \mathrm{D}$ and $2 \mathrm{~F}$ ) and axon commissures are thicker and frequently fused, providing additional support for a role for the WRC in midline repulsion. Taken together, these genetic interaction results strongly suggest that the WRC functions in SlitRobo1-mediated repulsive signaling at the midline.

The WIRS motif in Robo1 is important for its interaction with the WRC

The cytoplasmic tail of Robo1 contains a WIRS motif which is conserved in vertebrates (Figure 3A). The purified cytoplasmic tail of human Robo1 directly interacts with the WRC in pulldown assays via its WIRS motif (Chen et al., 2014a). To determine if this WIRS-dependent interaction of Robo1 with the WRC is conserved in Drosophila, we performed coimmunoprecipitation assays in Drosophila embryonic S2R+ cells (DGRC, \#150) using tagged constructs of Robo1 and HSPC300. We found that Robo1 immunoprecipitated with HSPC300 indicating that Drosophila Robo1 can also interact with the WRC (Figure 3C). Next, we introduced point 
mutations into the WIRS motif of Robo1 (Robo1 $\Delta$ WIRS; Figure 3B) and found a significant decrease in the amount of Robo1 that immunoprecipitated with HSPC300 (Figure 3C and 3E). Thus, mutating the WIRS motif substantially disrupts the binding of Robo1 to the WRC indicating that Robo1 interacts with the WRC partly via the WIRS motif. In contrast, the previously published interaction data for human Robo1 (Chen et al., 2014a) showed that mutating the WIRS motif completely abolishes binding to the WRC. We speculate that there may be a small amount of indirect binding of Robo1 to the WRC via Ena or DOCK which are known interactors of Robo1 (Bashaw et al., 2000; Fan et al., 2003). Previous work has identified interactions between Ena and Abi (Chen et al., 2014b) and between the DOCK homolog Nck and Nap1 (Kitamura et al., 1996). Both Abi and Nap1 are members of the WRC. As the pulldown assay with human Robo1 was done using purified proteins, any indirect binding will not be detected. Support for this notion comes from our coimmunoprecipitation results of Robo2 and HSPC300. Drosophila Robo2 is structurally similar to Robo1 except that it lacks the conserved cytoplasmic motifs CC2 and CC3 present in Robo1 that serve as the interaction sites for Ena and DOCK (Bashaw et al., 2000; Fan et al., 2003) (Figure S2A). Indeed, we find that Robo2 can also interact with HSPC300 though mutating the WIRS motif of Robo2 completely abolishes this interaction (Figure S2B and S2C). This result is consistent with the idea that there might be indirect binding of the WRC to Robo1 via its interaction with other WRC partners but not to Robo2 that lacks any such interactions.

Next, we wanted to test whether the Robo1-WRC interaction is regulated by the Robo ligand Slit. We treated S2R+ cells with bath application of Slit-conditioned media (CM) and found a substantial increase in the interaction between Robo1 and HSPC300 as compared to cells treated with control CM (Figure 3D and 3F). By contrast, Robo1 $\Delta$ WIRS shows no significant increase in binding to HSPC300 upon Slit CM treatment. As there is significant variability in the activity of Slit CM with each preparation, we see different levels of 
enhancement in binding obtained with each Slit treatment. Nevertheless, Slit application

consistently increases the interaction between Robo1 and HSPC300. These results suggest

that upon Slit binding, the WRC is recruited to Robo1 via its WIRS motif.
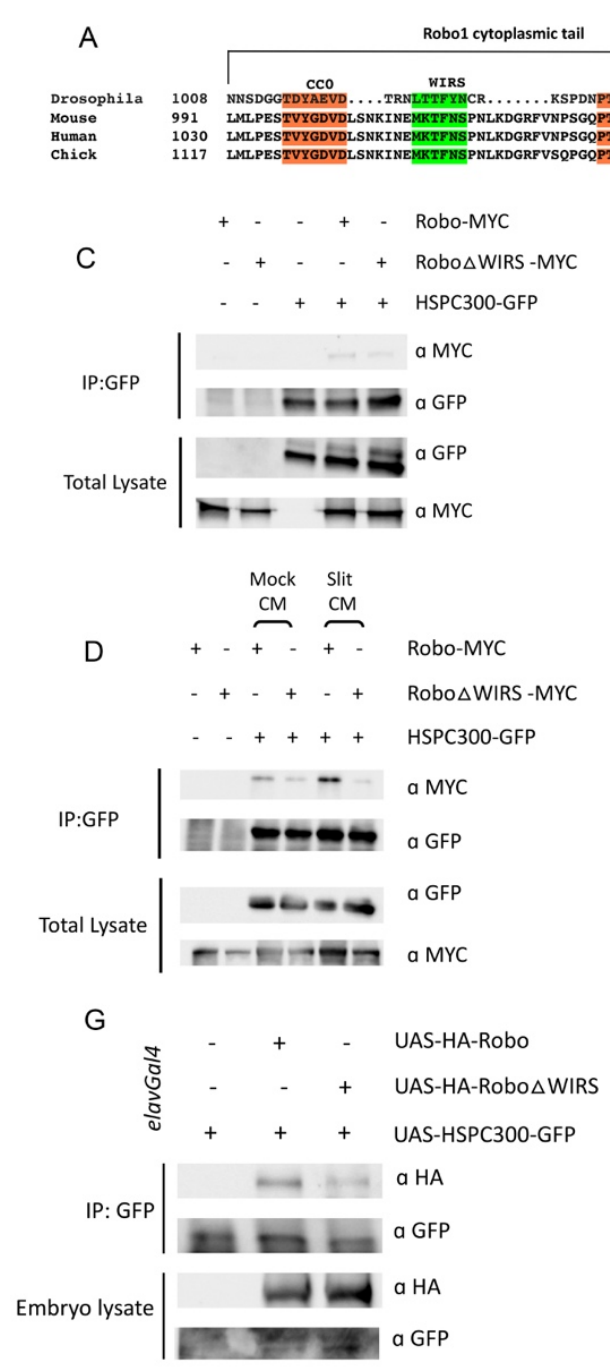

$\mathrm{E}$

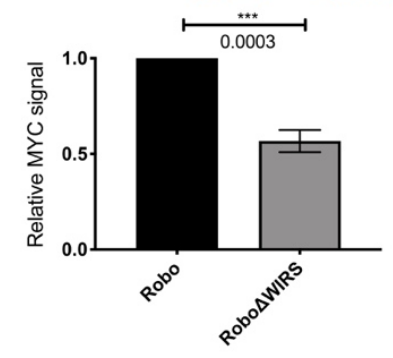

$\mathrm{F}$

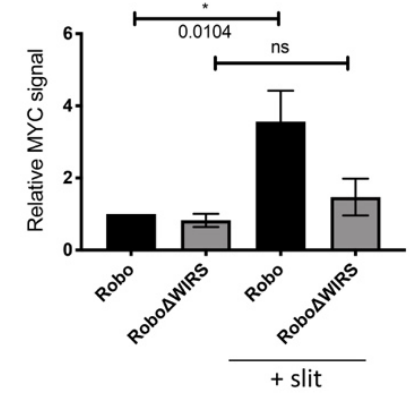

$\mathrm{H}$

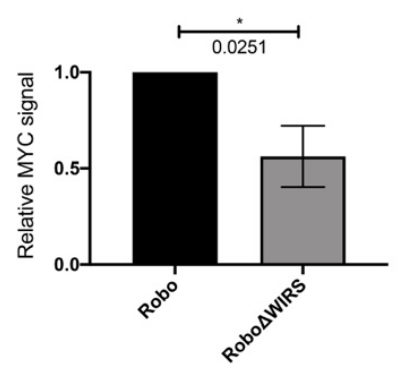

Figure 3. Slit-dependent recruitment of the WRC to Robo1 requires the WIRS motif. (A) Sequence alignments of the cytoplasmic tail of Robo1 showing that the WIRS motif is conserved across species. (B) Schematic depicting the residues of the WIRS motif that are mutated in the Robo1 $\triangle$ WIRS variant. (C) Drosophila S2R+ cell lysates co-expressing HSPC300-GFP with either wild type Robo1-MYC or Robo1 $\triangle$ WIRS-MYC were immunoprecipitated with an anti-GFP antibody. The first three lanes show the individual proteins expressed alone. The fourth lane shows wild type Robo1 co-immunoprecipitating with HSPC300 while the fifth lane shows that mutating the WIRS motif decreases this binding. (D) Cell lysates were immunoprecipitated with anti-GFP following a 12' bath application of mock conditioned media or conditioned media obtained from Slit-expressing cells. The interaction between wild type Robo1 and HSPC300 is increased in the presence of Slit however no significant increase is noted with Robo1 $\Delta$ WIRS. (E and F) Quantitative representations of band intensities of the MYC-tagged Robo1 
variants in the immunoprecipitates normalized to wild type Robo1-MYC. Data were normalized to lysate levels of the Robo1 variants and HSPC300 levels in the immunoprecipitates. Error bars represent SEM. Number of trials, $n=4$ (for $E$ ) and 5 (for F). Significance was assessed using Student's $t$-test (for $E$ ) and one-way ANOVA with Tukey's multiple comparisons test (for F). (G) Drosophila embryo lysates expressing HSPC300-GFP alone (lane 1), with wild type HA-Robo1 (lane 2) or HA-Robo1 $\triangle$ WIRS (lane 3) pan-neuronally using an elavGal4 driver, were immunoprecipitated with anti-GFP. Wild type Robo1 coimmunoprecipitates with HSPC300 and mutating the WIRS motif decreases this binding. $(\mathrm{H})$ Quantitative representations of band intensities of the HA-tagged Robo1 variants in the imuunoprecipitates normalized to wild type HA-Robo1. Data were normalized to the lysate levels of the Robo1 variants and HSPC300 levels in the immunoprecipitates. Error bars represent SEM. Number of trials, $n=5$. Significance was assessed using Student's $t$-test.

Finally, to test whether this interaction occurs in vivo, we performed co-immunoprecipitation assays using Drosophila embryonic protein lysates. We generated transgenic flies using the GFP-tagged HSPC300 construct and HA-tagged Robo1 constructs. The pan-neuronal elavGal4 driver was used to drive expression of UAS-HSPC300 alone or with the wild type UAS-HARobo1 or UAS-HA-Robo1 immunoprecipitates with HSPC300 while mutating the WIRS motif results in a significant decrease in this binding (Figure $3 \mathrm{G}$ and $3 \mathrm{H}$ ). These results indicate that Robo1 interacts with the WRC in vivo as well and that this interaction is partly dependent on the WIRS motif.

The WIRS motif is essential for Robo1 function in vivo

To test whether this interaction with the WRC is required for Robo1 function in vivo, we compared the gain-of-function and rescue phenotypes of wild type Robo1 and Robo1 $\Delta$ WIRS in specific neuronal subsets in the Drosophila ventral nerve cord. We generated transgenic flies with wild type UAS-Robo1 or UAS-Robo1 $\triangle W I R S$ constructs. Both the transgenes are tagged with an HA epitope and inserted into the same genomic locus. Immunostaining for HA shows that both transgenes are expressed at comparable levels (Figure 4D and 4E). Using the egGal4 driver we expressed these transgenes in eagle neurons, a subset of commissural neurons. Eagle neurons, visualized here using a GFP reporter, consist of two populations: the EG 
population, which extends its axons in the anterior commissure of a segment, and the EW population, which extends axons in the posterior commissure (Figure 4A).
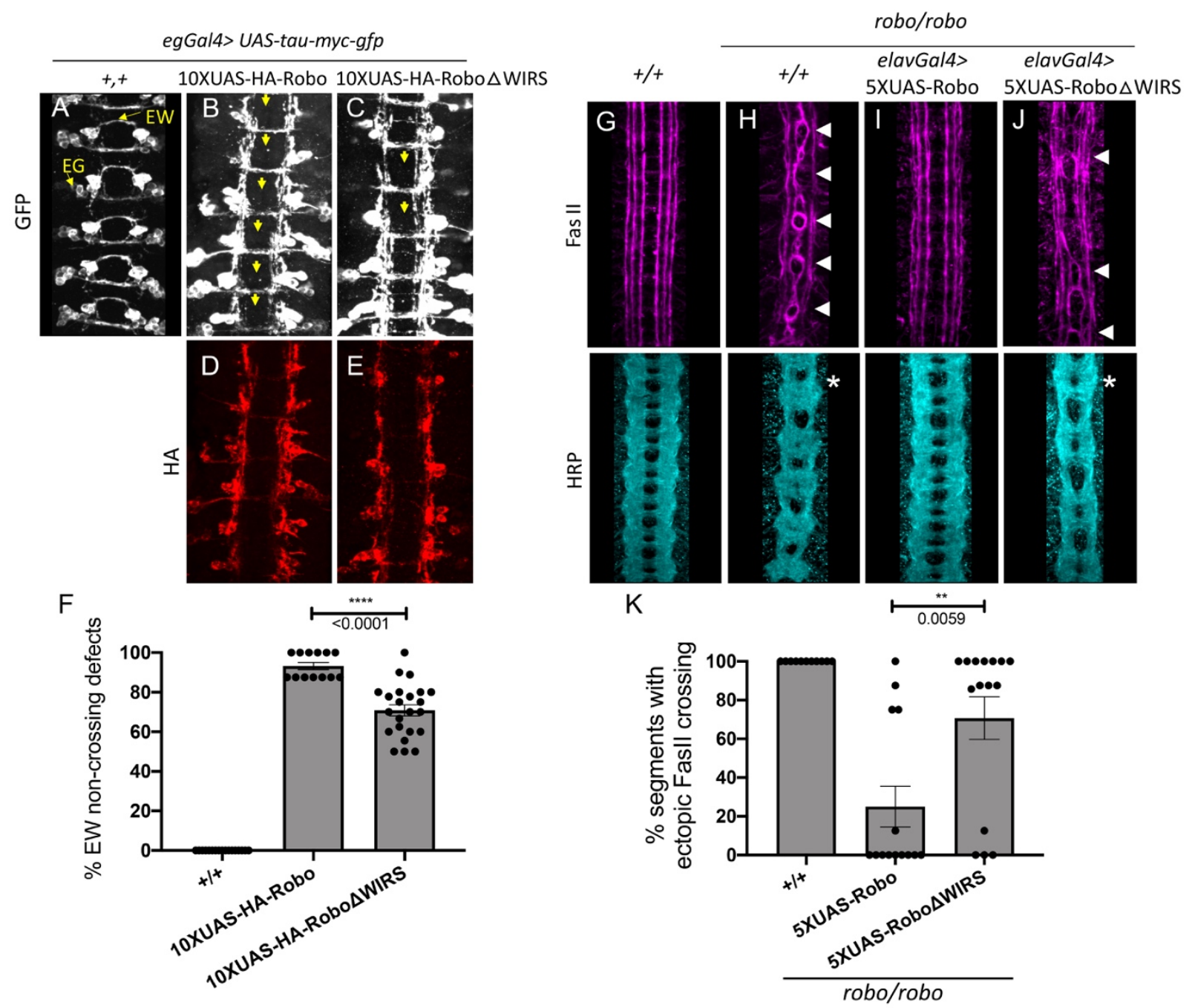

Figure 4. The WIRS motif is essential for Robo1 function in vivo. (A-C) Stage 16 Drosophila embryos carrying eg-Gal4 and UAS-TauMycGFP transgenes stained with anti-GFP which labels cell bodies and axons of the eagle neurons ( $E G$ and $E W$ ) in these embryos. EG neurons project through the anterior commissure of each segment while EW neurons project through the posterior commissure. (A) EW neurons cross in $100 \%$ of segments in wild type embryos. (B) Misexpression of wild type HA-Robo1 in eagle neurons results in a strong disruption of midline crossing where EW axons fail to cross in almost all segments of the nerve cord (yellow arrows). (C) Misexpressing HA-Robo1 $\Delta$ WIRS results in a significantly milder disruption with fewer segments showing EW non-crossing defects. (D and E) Embryos stained with anti-HA show comparable expression of the HA-tagged Robo1 variants. (F) Quantitation shows the percentage of segments in which EW axons fail to cross the midline. Data are presented as mean \pm SEM, number of embryos, $n=17,13,23$. Significance was assessed using Student's $t$-test. (G-J) Stage 17 embryos stained with anti-Fasll and anti-HRP. (G) Wild type embryos show no ectopic Fasll crossing defects and no phenotype in HRP. (H) robo mutants show severe ectopic Fasll crossing defects in $100 \%$ of segments (arrowheads) and a strong HRP phenotype with thickening and fusion of commissures (asterisk). (I) Pan-neuronal expression of wild type 5XUAS-Robo1 significantly rescues the robo mutant 
phenotype in Fasll as well as HRP however (J) 5XUAS-Robo1 $\triangle W I R S$ fails to rescue the robo mutant phenotype as efficiently as wild type Robo1 with frequent ectopic crossing in Fasll and thickened commissures in HRP still evident in these embryos. (K) Quantitation shows the percentage of segments in which Fasll axons ectopically cross the midline. Data are presented as mean $\pm \mathrm{SEM}$, number of embryos, $n=11,14,15$. Significance was assessed using Student's $t$-test.

Overexpression of wild type Robo1 in these neurons causes ectopic repulsion from the midline, resulting in a strong gain-of-function phenotype where almost all EW axons fail to cross the midline (Figure 4B). In contrast, overexpression of Robo1 $\Delta \mathrm{WIRS}$ results in a significantly weaker gain-of-function phenotype where EW axons in approximately $70 \%$ of segments fail to cross the midline (Figure 4C and 4F). Thus, mutating the WRC interaction site on Robo1 hampers its ability to induce ectopic repulsion in vivo.

Next, we assessed the ability of Robo1 $1 \mathrm{WIRS}$ to rescue the ectopic crossing defects of Fasll-positive axons seen in robo1 mutant embryos. Unlike in wild type embryos, where Fasll axons never cross the midline (Figure 4G), in robo1 mutants, axons in the medial most fascicle freely cross and recross the midline in $100 \%$ of segments (Figure $4 \mathrm{H}$ ). Re-expressing wild type Robo1 with the pan-neuronal driver elav-Gal4 restores the ipsilateral projection pattern in most of the segments, lowering the frequency of ectopic crossing to $25 \%$ of segments (Figure $4 \mathrm{I}$ ). In contrast, re-expression of Robo1 $1 \mathrm{WIRS}$ fails to rescue the crossing defects in $70 \%$ of segments (Figure $4 \mathrm{~J}$ and $4 \mathrm{~K}$ ). This indicates that in the absence of a functional WIRS motif, Robo1 is not nearly as effective at restoring repulsive signaling in ipsilateral axons in vivo. Altogether, these results suggest a role for the WIRS motif in Robo1 repulsive signaling at the midline.

Mutating the endogenous WIRS motif disrupts Robo1 function in vivo

Our in vivo results obtained so far have relied on misexpression or overexpression of Robo1 that likely is not subject to the adequate spatial and temporal regulation that is critical for guidance receptor function. Further, such unregulated high levels of Robo1 expression on the 
cell surface could potentially mask subtle dysfunction in receptor activity. We see this especially for the rescue experiments with our UAS-Robo1 transgenes. While the difference in rescue ability between 5XUAS-Robo1 and 5XUAS-Robo1 $\triangle W I R S$ is around 50\% (Figure 4K), performing this rescue assay with 10XUAS-Robo1 and 10XUAS-Robo1 $\triangle W I R S$ transgenes, which have double the number of UAS enhancer sites and express much higher levels of the Robo1 variants, gives a much more modest difference of $13 \%$ (Figure S3A-S3E). Indeed, in rescue experiments using 10XUAS-Robo1 transgenes, we see strong gain of function effects that lead to both rescue of abnormal crossing of Fasll positive axons, as well as ectopic repulsion of commissural axons (Figure S3F-S3J). Given these caveats, we sought to analyze the function of the WIRS motif in Robo1 signaling in a more endogenous context. First, we performed a rescue assay with an HA-tagged genomic rescue construct of robo 1 that contains upstream and downstream regulatory regions of Robo1 in addition to the Robo1 coding sequence (Brown et al., 2015). Transgenics created with this construct show a Robo1 expression pattern that closely resembles that of endogenous Robo1 (Brown et al., 2015). We mutated the WIRS motif in this robo1 genomic rescue construct and inserted the transgene into the same genomic site as the wild type construct. Both transgenes show comparable levels of Robo1 expression upon HA immunostaining (Figure S4A and S4B). We tested the ability of these transgenes to rescue the robo1 mutant phenotype in Fasll-positive axons. One copy of the wild type robo1 genomic rescue construct $\left(r o b o^{\text {gen }}\right)$ was able to rescue ectopic crossing of Fasll-positive axons in almost all segments with only $6 \%$ still showing defects (Figure $5 \mathrm{~A}$ ) while robo1 $\triangle W I R S$ genomic rescue construct (robo1 $\left.\triangle W I R S^{g e n}\right)$ was unable to rescue ectopic crossing defects in over $70 \%$ of segments (Figure 5B and 5C). Similarly, for HRP stained axons, the frequent thickening and fusion of the anterior and posterior commissures in robo1 mutants can be rescued with the wild type robo1 ${ }^{\text {gen }}$ but not with robo1 $\triangle W I R S^{g e n}$ (Figure $5 A$ and $5 B$ ). These 
results, in more physiologically relevant contexts, demonstrate a marked decline in Robo1

function upon disruption of the WRC binding site.
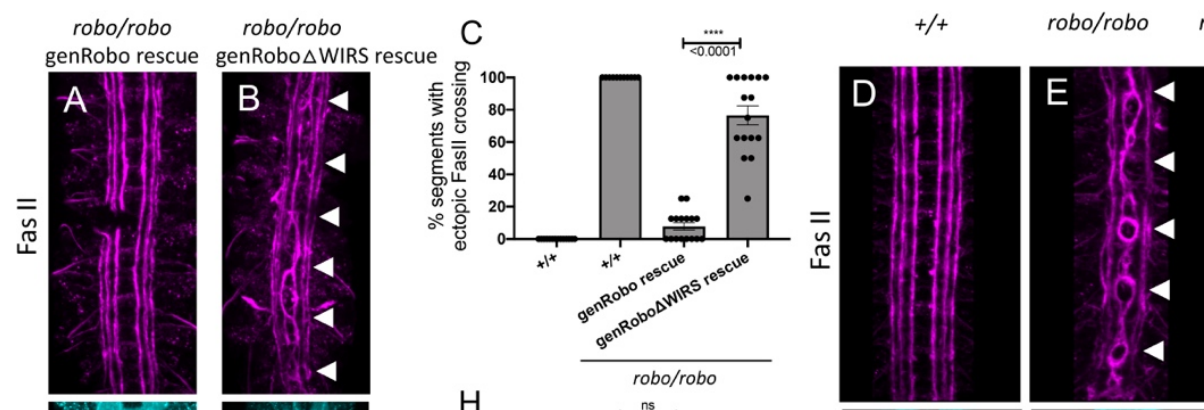

robo $\triangle$ WIRS CRISPR robo $\triangle$ WIRS CRISPR
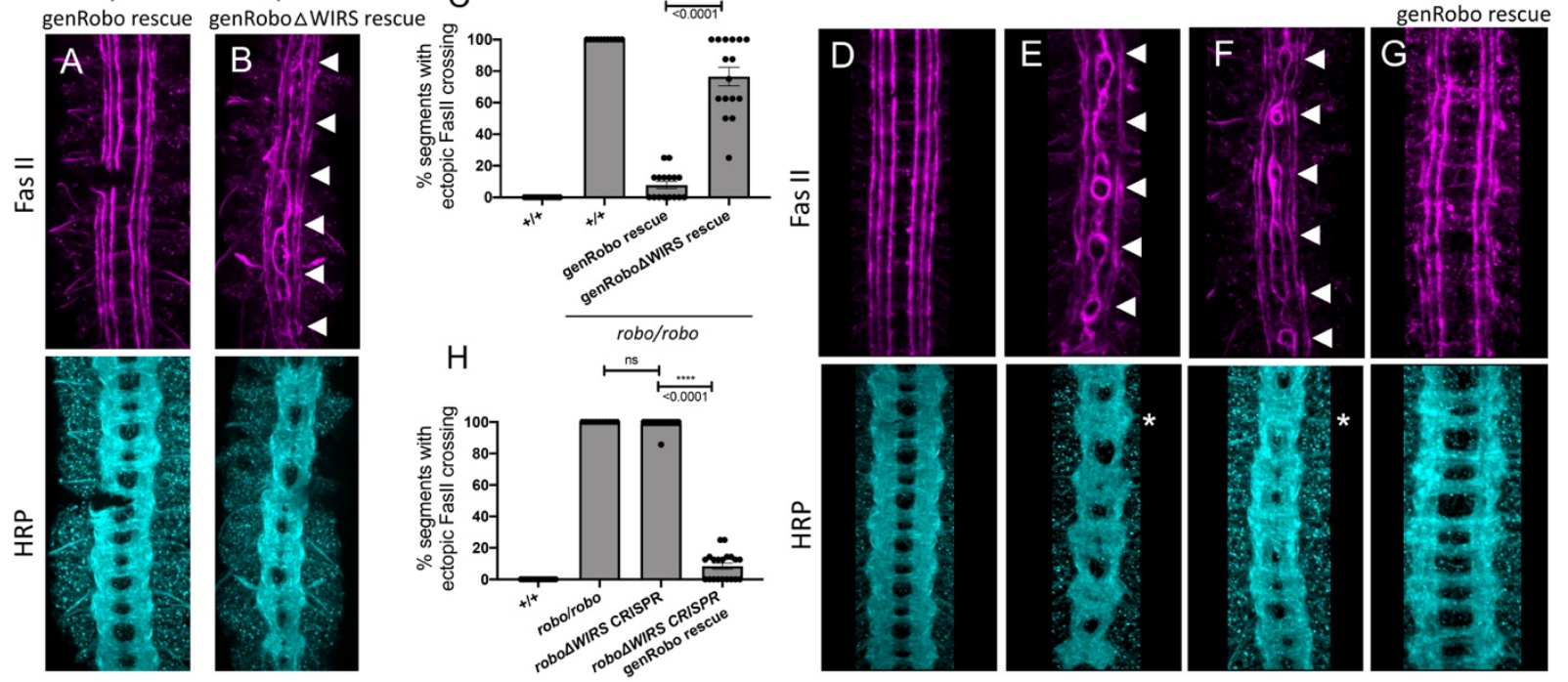

Figure 5. Mutating the endogenous WIRS motif disrupts Robo1 function in vivo. (A and B)

Stage 17 embryos stained with anti-Fasll and anti-HRP. (A) The strong Fasll and HRP phenotypes seen in robo mutant embryos can be completely rescued with a wild type genomic Robo1 rescue construct (genRobo) that contains additional upstream and downstream regulatory regions of robo1, more closely mimicking the endogenous Robo1 expression pattern. (B) In contrast, the genomic Robo1 rescue construct containing mutations in the WIRS motif of Robo1 (genRobo $\Delta$ WIRS) fails to rescue the robo mutant phenotype in both Fasll and HRP. (C) Quantitation shows the percentage of segments in which Fasll axons ectopically cross the midline. Data are presented as mean $\pm S E M$, number of embryos, $n=$ $14,11,16,16$. Significance was assessed using Student's $t$-test. (D-G) Stage 17 embryos stained with anti-Fasll and anti-HRP. (D) Wild type embryos showing no phenotype in Fasll or HRP. (E) robo mutants show severe ectopic Fasll crossing defects in $100 \%$ of segments (arrowheads) and a strong HRP phenotype with thickening and fusion of commissures (asterisk). (F) CRISPR embryos with mutations in the endogenous WIRS motif show severe phenotypes in Fasll and HRP bearing strong resemblance to robo mutants. (G) The phenotypes seen in these CRISPR robo $\triangle$ WIRS embryos can be completely rescued with the wild type genomic Robo1 rescue construct. (H) Quantitation shows the percentage of segments in which Fasll axons ectopically cross the midline. Data are presented as mean $\pm \mathrm{SEM}$, number of embryos, $n=14,11,14,20$. Significance was assessed using one-way ANOVA with Tukey's multiple comparisons test.

Finally, using the CRISPR-Cas9 system, we mutated the WIRS motif in the endogenous robo1

locus. We used a single guide RNA that targets the endogenous WIRS motif and a single-

stranded oligonucleotide template to introduce point mutations in the WIRS motif (Figure S5A).

We sequenced the regions surrounding the WIRS motif to verify that we had successfully 
mutated the WIRS motif without introducing any unwanted frameshift mutations or deletions. While we found no frameshifts, we did notice that our strategy had resulted in an unexplained loss of the smaller intron 16 (Figure S5A). Since the robo $1^{\text {gen }}$ constructs and the previously used robo swap alleles (Spitzweck et al., 2010) that can restore Robo1 function fully, do not contain any intronic sequences, we believe that it is extremely unlikely that the loss of this intron affects Robo1 function. Next, we analyzed the phenotypes of both HRP and Fasll-positive axons in these robo1 $\triangle W I R S$ CRISPR embryos. We see a surprisingly strong ectopic crossing phenotype in these embryos with defects in almost $100 \%$ of segments, showing that they fully phenocopy the robo1 mutant embryos (Figure 5D-5F). We were able to achieve a near perfect rescue with the introduction of one copy of robo $1^{\text {gen }}$ indicating that this phenotype is not a result of any off-target effects arising from Cas9-mediated cleavage (Figure $5 \mathrm{G}$ and $5 \mathrm{H}$ ). This result also supports our interpretation that the loss of intron 16 in our CRISPR allele has no effect on Robo1 function, since the robo $1^{\text {gen }}$ construct does not include any introns. As an additional control, we also tested whether the robo1 $\triangle W I R S$ CRISPR mutations disrupt normal Robo1 expression. To investigate this, we immunostained for Robo1 expression using a monoclonal Robo1 antibody. Unlike the robo1 mutants in which no Robo1 protein can be detected (Figure S5D and S5G), we see substantial Robo1 staining in the robo1 $\triangle W I R S$ CRISPR mutants suggesting that the phenotype is not due to a failure in protein production (Figure S5C and S5F). Altogether, these results strongly suggest a critical role for the WIRS motif in Robo1 repulsive function in vivo.

The WIRS motif is required for Slit-dependent repulsion in mouse spinal commissural axons

The WIRS motif in the Robo1 receptor is conserved in vertebrates, raising the possibility for a potential role in vertebrate Robo1 signaling. Indeed, the cytoplasmic tail of human Robo1 can 
bind to the WRC via its WIRS motif (Chen et al., 2014a). Thus, to address the question of whether the WIRS motif is important for vertebrate Robo1 signaling, we introduced point mutations into the WIRS motif of human Robo1 (hRobo1 $\Delta \mathrm{WIRS}$ ) and performed gain-of-function experiments with wild type hRobo1 and hRobo1 $\Delta \mathrm{WIRS}$ constructs. We electroporated E12 mouse spinal cords with wild type hRobo1 or hRobo1 $\Delta \mathrm{WIRS}$, along with RFP to visualize electroporated neurons and cultured dorsal spinal cord explants next to mock 293T cell aggregates or cell aggregates expressing Slit (Figure 6A). Explants cultured adjacent to mock cell aggregates show uniform outgrowth on all sides of the explant (Figure 6B). In contrast, explants cultured adjacent to Slit-expressing aggregates show decreased outgrowth on the side proximal to the Slit-expressing aggregate as compared to the distal side (Figure 6C). Explants electroporated with wild type hRobo1 show an increased repulsive response to Slit with even less outgrowth on the proximal side and a significantly lower proximal/distal outgrowth ratio (Figure 6D and 6F). In contrast, explants electroporated with hRobo1 $\Delta$ WIRS show no such gain of function response to Slit and have a proximal/distal outgrowth ratio similar to that seen for RFP electroporated explants (Figure 6E and 6F), suggesting that the WIRS motif is important for the Slit-induced repulsive response of vertebrate Robo1. Next, to assess whether the WIRS motif is also important for the collapsing activity of Robo1 in response to Slit, we performed Slitinduced collapse assays using dissociated E12 mouse dorsal spinal commissural neurons (Figure $6 \mathrm{G}$ and $6 \mathrm{H}$ ). In our control cultures, 38\% of Robo3-positive commissural axons show collapsed growth cones (Figure 6I). Following a 30-minute treatment with recombinant Slit2, we see an increase in the collapse rate to $62 \%$. Neurons electroporated with wild type MYC-tagged hRobo1 show a further increase in collapse rate with $77 \%$ of Robo3- and MYC-positive axons ending in collapsed growth cones. In contrast, we saw no increase in the number of collapsed growth cones in neurons electroporated with MYC-tagged hRobo1 $\triangle \mathrm{WIRS}$ (Figure 6I) suggesting that the WIRS motif is also important for the Slit-induced collapsing activity of Robo1. 

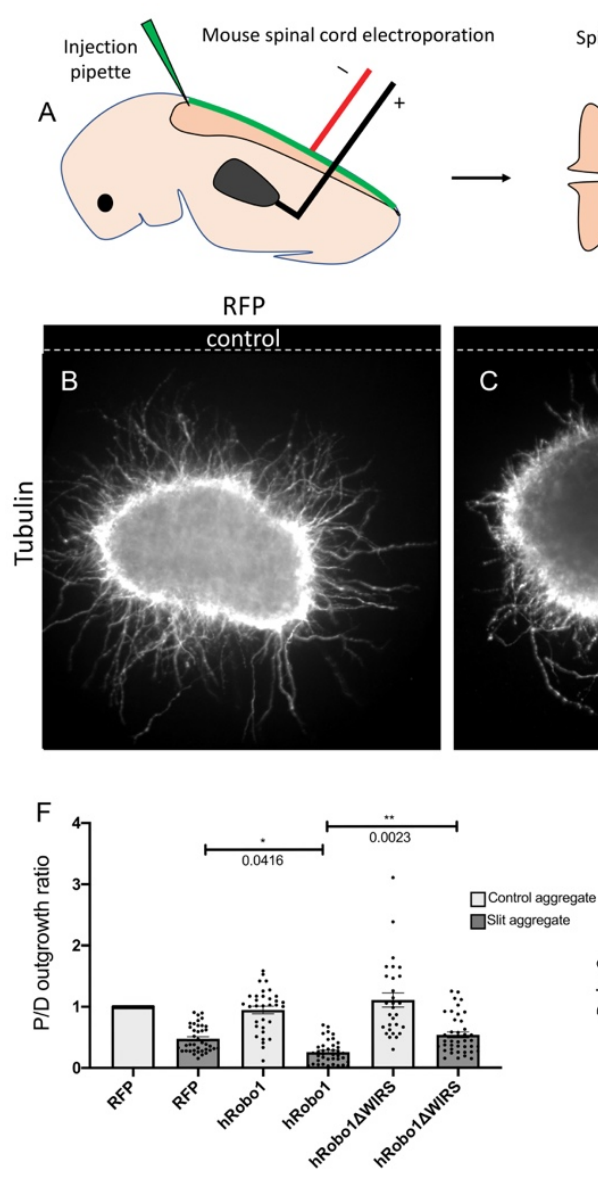

Spinal cord dissection

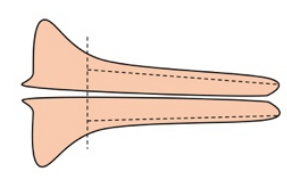

RFP

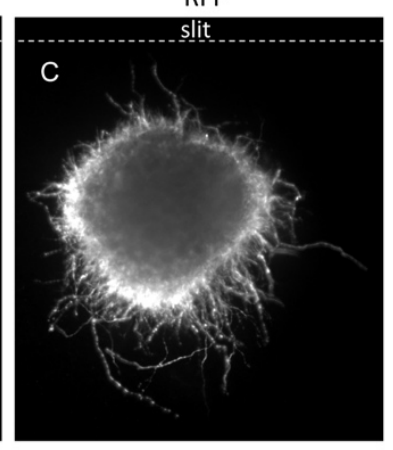

Control

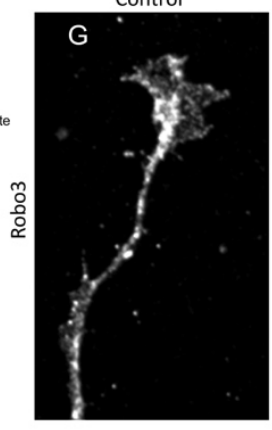

Preparation of dorsal spinal cord explants
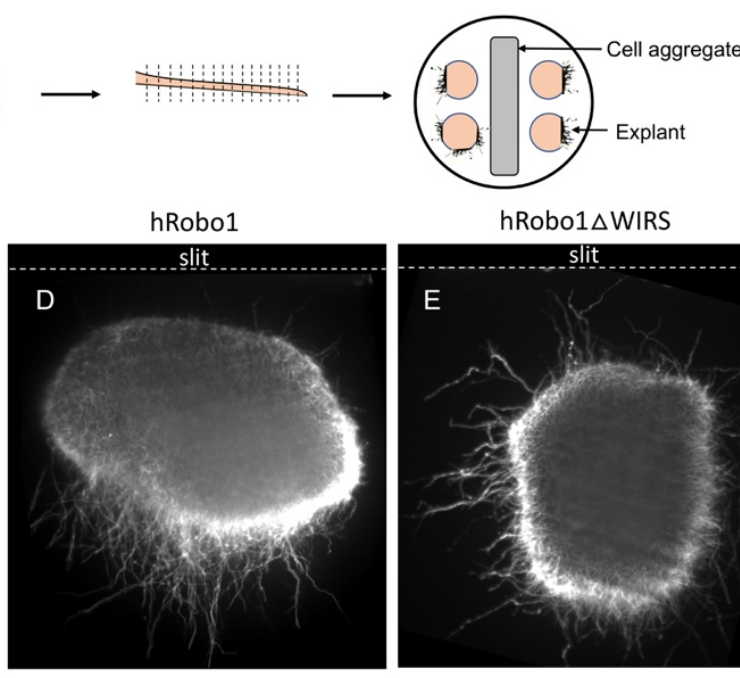

Slit

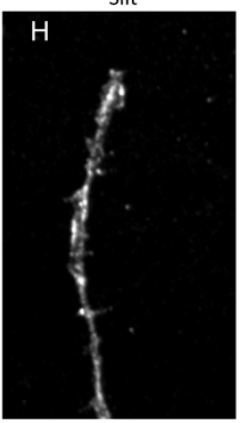

Explant outgrowth assay
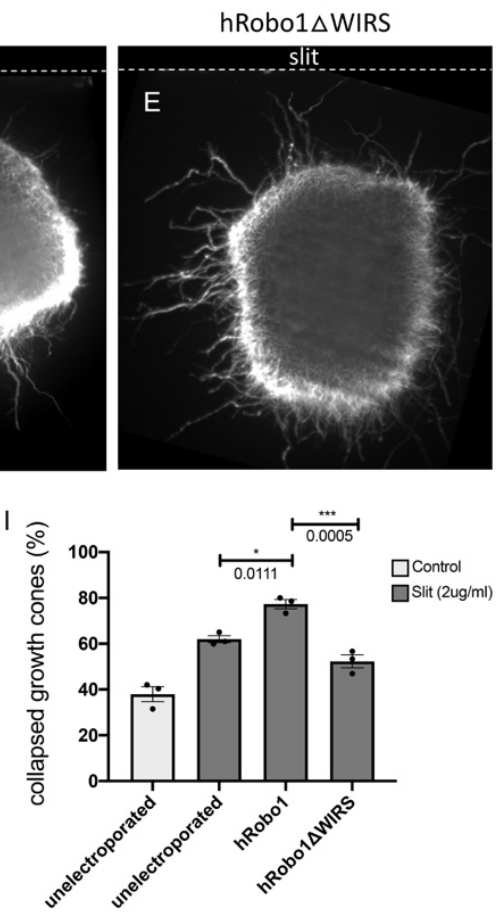

Figure 6. The WIRS motif is required for Slit-dependent repulsion in mouse spinal commissural axons. (A) Schematic of electroporation and culture of spinal cord explants. Dotted lines show cut sites to obtain dorsal spinal cord explants. The image on the right depicts the arrangement of explants cultured around a 293T cell aggregate (control or Slit-expressing) embedded in collagen. (B-E) E12 dorsal spinal cord explants labeled with anti-Tubulin to visualize axon outgrowth. Dotted lines indicate position of the cell aggregate. (B) RFP electroporated explant cultured next to a mock cell aggregate shows uniform outgrowth on all sides of the explant. (C) RFP electroporated explant cultured next to a Slit-expressing cell aggregate shows decreased outgrowth on the side proximal to the aggregate as compared to the side distal to it. (D) Explant electroporated with wild type hRobo1 cultured next to a Slit-expressing cell aggregate shows even less outgrowth on the proximal side demonstrating increased responsiveness to Slit. (E) Explant electroporated with hRobo1 $\Delta$ WIRS cultured next to a Slit-expressing cell aggregate shows no such increase in Slit responsiveness as the proximal: distal outgrowth ratio is similar to that seen for RFP electroporated explants. (F) Quantification shows the proximal:distal outgrowth ratio for explants cultured next to control cell aggregates (white) and Slitexpressing cell aggregates (grey). Data are presented as mean \pm SEM, number of explants, $n=29,39,33$, $39,29,41$ (from 3 independent experiments). Significance was assessed using one-way ANOVA with Tukey's multiple comparisons test. ( $\mathbf{G}$ and $\mathbf{H})$ Growth cone collapse in response to Slit in E12 dissociated commissural axons. Growth cone morphology was examined by staining for the commissural marker Robo3. (I) Quantification shows percentage of axons with collapsed growth cones. Unelectroporated neurons show an increased level of collapse when treated with Slit. Neurons electroporated with wild type hRobo1 show a gain-of-function response to Slit with an even higher collapse level. In contrast, 
neurons electroporated with hRobo1 $\Delta$ WIRS show no gain-of-function and a collapse level similar to unelectroporated neurons. For neurons electroporated with the MYC-tagged hRobo1 variants, only Robo3- and MYC- positive axons were analyzed. Data are presented as mean $\pm S E M$, number of trials, $n$ $=3$ (over 30 neurons for each condition/trial). Significance was assessed using one-way ANOVA with Tukey's multiple comparisons test.

The hRobo1 variants show comparable levels of MYC staining in neurons (Figure S6A-S6D). Together, these results from mouse dorsal spinal cord explants and dissociated neuron cultures show that while overexpression of wild type hRobo1 is able to enhance the repulsive response to Slit, mutating the WIRS motif in hRobo1 abolishes this gain-of-function response, indicating that the WIRS motif is important for vertebrate Robo1 signaling as well and suggest an evolutionarily conserved role for the WIRS motif in Robo1 repulsive signaling.

\section{DISCUSSION}

In this manuscript, we have documented a conserved role for the WRC in Slit-mediated Robo1 repulsive signaling. Using the developing Drosophila embryonic CNS, we demonstrate a series of dose-dependent genetic interactions between components of the WRC and Slit-Robo1 signaling which suggest that the WRC functions in vivo to regulate Robo1 repulsive signaling at the midline. Biochemical experiments in cultured cells show that Robo1 can bind to the WRC partially via its WIRS motif and that Slit stimulation can induce recruitment of the WRC to Robo1. Further, we present several lines of evidence to show that the WIRS motif is important for Robo1 function in vivo. First, mutating the WIRS motif results in a significantly weaker gainof-function phenotype when Robo1 is misexpressed in commissural axons. Second, the Robo1 variant with mutations in its WIRS motif, fails to rescue the robo1 mutant phenotype as effectively as wild type Robo1. Finally, mutating the WIRS motif in the endogenous robo1 locus using the CRISPR-Cas9 system results in embryos with severe ectopic crossing defects that phenocopy robo1 mutants. These data demonstrate a severe decline in Robo1 function upon disruption of the WRC binding site. We also present several lines of evidence that support an 
evolutionarily conserved role for the WIRS motif in vertebrate Robo1 signaling. First, we show that Robo1 $\triangle$ WIRS is less effective at mediating repulsion in response to Slit in explants from the mouse dorsal spinal cord. In addition, Robo1 $\Delta$ WIRS is less responsive to the collapsing activity of Slit in dissociated spinal commissural axons. Together, our observations support the model that Slit stimulation results in recruitment of the WRC to the WIRS motif in Robo1 which is vital to Robo1-mediated repulsive signaling at the midline (Figure 7).

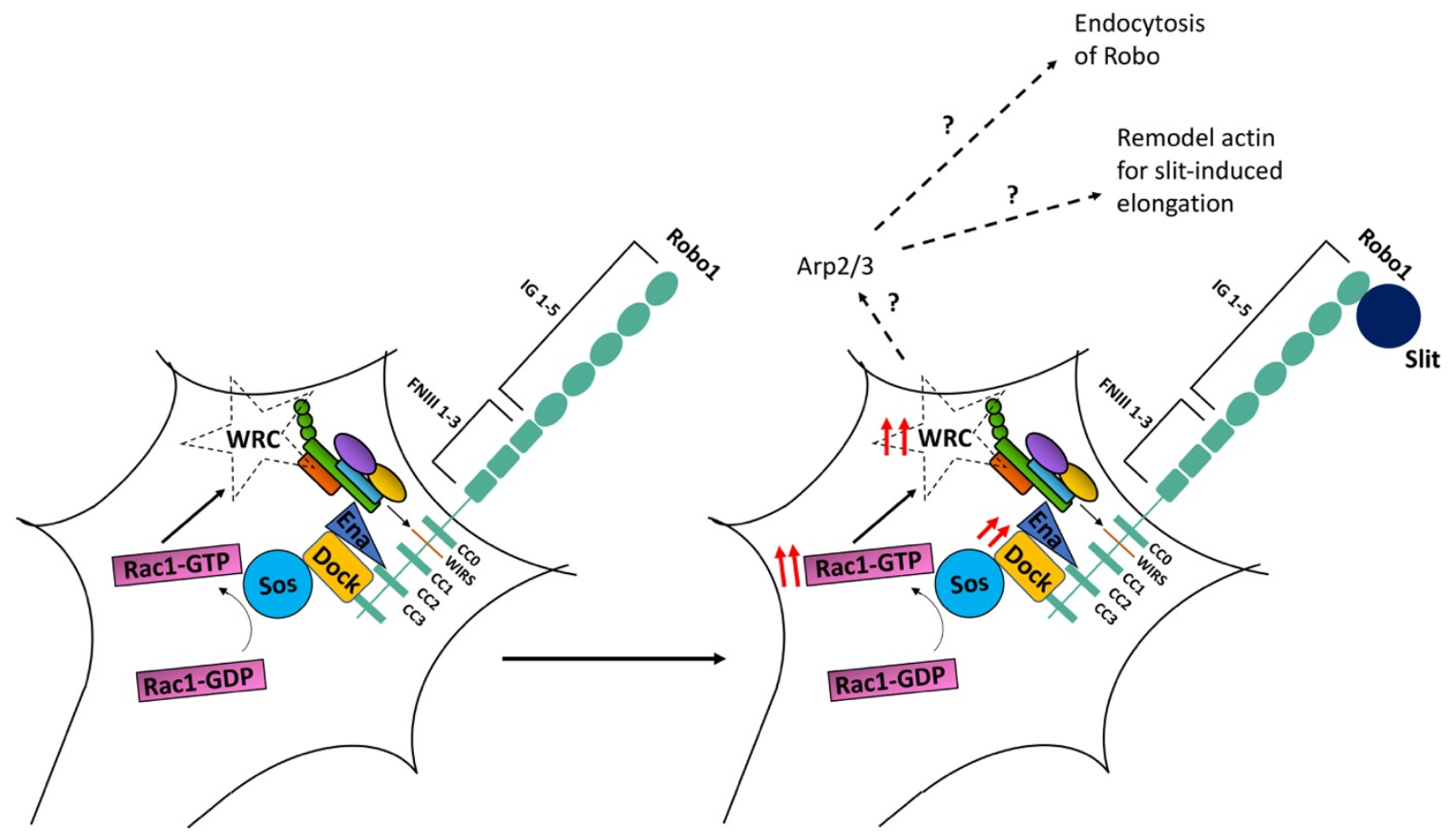

Figure 7. A model of WRC function in Robo1 signaling. In our proposed model, the WRC binds to Robo1 partly via its WIRS motif. Rac1 is activated downstream of Robo1 (Fan et al., 2003; Wong et al., 2001) which likely activates the complex. Slit binding induces increased WIRS-dependent recruitment of the WRC to Robo1 which is vital to Robo1-mediated repulsive signaling. We speculate that the WRC functions downstream of Robo1 by activating Arp2/3 to remodel the actin cytoskeleton in order to facilitate either Robo1 endocytosis or an initial extension of Slit-induced filopodia.

In this study, we used a series of complementary approaches to evaluate the importance of the WIRS motif for Robo1 repulsive signaling. While it is generally assumed that the high expression levels resulting from the Gal4/UAS system are unlikely to reflect normal spatial and temporal regulation, it remains unclear to what extent this might confound comparisons between 
different mutant variants of a given protein. Our results with Robo1 indicate that Gal4UAS/directed expression significantly hinders the detection of critical structural elements of the receptor. For example, when using a pan-neuronal driver to reintroduce Robo1 into the robo1 mutant embryos, we observe only very modest differences between the wild type and WIRS mutant forms of overexpressed Robo1. In contrast, we see much more severe phenotypes when the WIRS motif is disrupted under conditions that more closely match the endogenous robo1 levels using the robo1 genomic rescue constructs or when mutating the WIRS motif in the endogenous robo1 locus using the CRISPR-Cas9 system. These direct comparisons between different assays to measure protein function suggest that rescue experiments using the Gal4/UAS system must be interpreted with caution. This also suggests that our results comparing the gain of function effects of Robo1 and Robo1 $1 \mathrm{WIRS}$ in vertebrate systems are likely to underestimate the significance of the WIRS motif and recruitment of the WRC for Robo1 repulsion.

Here, we have shown that the WRC is an important component of the Slit-Robo1 repulsive pathway at the midline. But what happens once the WRC is recruited to Robo1? Is the WRC acting via Arp2/3 to promote branched actin polymerization downstream of Robo1? The VCA region of Scar/Wave is sequestered within the complex until activation, which triggers a conformational change releasing the VCA domain (Chen et al., 2010; Ismail et al., 2009). Rac1 is an important activator of the WRC and has been previously found to be activated downstream of Robo1 in both Drosophila and mouse (Fan et al., 2003; Wong et al., 2001). We can speculate that this increased Rac1 activity is responsible for the activation of WRC upon Slit stimulation. Once active, the WRC can induce Arp2/3-mediated actin polymerization. At first glance, the initiation of actin polymerization downstream of Robo1 might seem paradoxical, however, many repulsive guidance cues recruit downstream effectors that enhance actin polymerization. One recent study demonstrates that dorsal root ganglion neurons initially extend filopodia toward a source of Slit before retracting (McConnell et al., 2016). This study highlights the nuanced and 
complex actin rearrangements that occur downstream of guidance cues, which potentially contribute to sensing of the environment for improved resolution of a guidance gradient. The WRC might play an important role in the generation of these Slit-induced filopodia by initiating the formation of branched actin filaments that are subsequently rebundled to form filopodia as suggested by the convergent elongation model that supports a role for Arp2/3 in filopodia formation in neurons (Yang and Svitkina, 2011). Ena/VASP proteins, downstream effectors of Robo1, are important for these Slit-induced filopodial extensions (McConnell et al., 2016) and on account of their actin bundling activity are perfectly poised to orchestrate this actin reorganization in order to drive filopodia formation. The WRC has also been shown to be important for other actin-dependent processes such as receptor endocytosis (Basquin et al., 2015; Xu et al., 2016). We have previously shown that endocytosis of Drosophila Robo1 upon Slit stimulation is essential for Robo1 repulsive signaling (Chance and Bashaw, 2015). It was recently reported that vertebrate Robo1 also undergoes endocytosis and recycling following Slit stimulation (Kinoshita-Kawada et al., 2019) suggesting a conservation of this regulatory mechanism. Future studies are needed to decipher how exactly the WRC contributes to Robo1 signaling either by influencing local actin remodeling for the initial advancement of growth cone extensions or by regulating Robo1 endocytosis and recycling to the plasma membrane. Additionally, other known downstream effectors of Robo1 like Ena and Abl have also been shown to influence WRC activity (Chen et al., 2014b; Leng et al., 2005). It would thus be interesting to dissect how this inter-regulation between these different components of Robo1 signaling contributes to fine-tuning of WRC activity to generate a specific output for Slit-Robo1 repulsion.

Previously, the WIRS motif has been shown to be important in Neuroligins and Syg-1 for proper synapse formation (Chia et al., 2014; Xing et al., 2018) as well as in Neogenin for the maintenance of adherens junctions (Lee et al., 2016). In these contexts, it is apparent that the WRC reinforces the F-actin network at these membrane junctions. However, it is unclear if the 
WIRS-WRC interactions are subject to regulation by the respective ligands or if the WRC performs more of a scaffolding function. In the context of axon guidance, our work demonstrates a ligand-dependent recruitment of the WRC to the WIRS domain of Robo1 suggestive of both spatial and temporal specificity of WRC activation. To our knowledge, this study is the first to demonstrate that the WRC can be recruited to a guidance receptor in response to a ligand.

In addition to functioning as part of the actin-regulating complex, WRC members can have functions independent of the complex as well. For example, CYFIP proteins can interact with fragile $\mathrm{X}$ mental retardation protein (FMRP) to regulate mRNA localization and protein translation (Abekhoukh and Bardoni, 2014; Schenck et al., 2003; Schenck et al., 2001) and Abi can interact with WASP and Diaphanous to regulate F-actin (Bogdan et al., 2005; Ryu et al., 2009). While we cannot entirely rule out a role for WRC-independent functions of these proteins in Robo1 signaling, several lines of evidence point to the involvement of the WRC as a whole downstream of Robo1. First, two separate WRC members, cyfip and $h s c p 300$, show genetic interactions with the Slit-Robo pathway. Second, the physical interaction between Robo1 and HSPC300 is dependent on the WIRS motif which requires a binding interface formed by CYFIP and Abi, that comes together only in the fully assembled WRC (Chen et al., 2014a). Finally, the strong midline crossing phenotypes we see upon manipulating the WIRS motif suggests that it is indeed this interaction with the fully assembled WRC that is important for Robo1 signaling in vivo.

Drosophila Robo1 has numerous functions in development outside of its role in midline repulsion and the robo1 $\triangle W I R S$ CRISPR mutants generated here also provide an opportunity to discern which developmental functions of Robo1 require the WRC in future studies. Robo1 regulates the migration of chordotonal sensory neurons (Kraut and Zinn, 2004) and mesodermal migration for muscle patterning (Kramer et al., 2001). Embryos lacking robo1 show defects in heart lumen formation (Qian et al., 2005) and tracheal migration (Englund et al., 2002). In mammals, Robo1 also plays important roles outside of the nervous system, including the 
formation of blood vessels (Rama et al., 2015) and organs like the heart (Mommersteeg et al., 2013) and the mammary glands (Macias et al., 2011), and it can also regulate stem cell proliferation (Ballard et al., 2015). Finally, the Slit-Robo pathway has been shown to regulate tumor angiogenesis along with tumor cell migration and metastasis (Tong et al., 2019). Misregulation of Slit-Robo signaling has been implicated in multiple types of tumorigenesis making it a promising target for cancer treatments (Koohini et al., 2019). Such therapeutic avenues require a comprehensive understanding of Slit-Robo signaling in specific cancers highlighting the importance of investigating the WRC as a downstream effector of Robo in disease contexts as well.

In addition to Robo1, other Robo receptors also contain WIRS motifs in their cytoplasmic domains. Drosophila Robo2 plays a minor role in midline repulsion and together with Robo3 also regulates lateral positioning of the longitudinal fascicles (Evans and Bashaw, 2010; Rajagopalan et al., 2000). As Robo2 and Robo3 do not contain CC2 and CC3 domains, to which most of the known Robo1 effectors bind, very little is known about their downstream signaling. Vertebrate Robo3 can induce repulsive signaling in response to a recently identified ligand, NELL2 (Jaworski et al., 2015) Vertebrate Robo3 also contains a WIRS motif in its cytoplasmic domain, raising the possibility that these Robo receptors could share a common cellular mechanism for repulsion, despite responding to distinct ligands. Additionally, attractive axon guidance receptors like Fra and its vertebrate ortholog DCC, also contain WIRS motifs in their cytoplasmic domains. Unsurprisingly, many core actin modifying proteins that act downstream of repulsive cues like Ena/VASP and Abl kinase, also function in attractive signaling (Forsthoefel et al., 2005; Gitai et al., 2003). We can speculate that the WRC might also function in both repulsion and attraction by regulating different actin-based processes like membrane trafficking versus growth cone advancement. Alternatively, as other studies have shown the importance of an initial growth cone extension toward repulsive cues, it is likely that tight spatiotemporal activation along with regulation by other effector molecules can result in 
fine-tuning of WRC activity to contribute to distinct cytoskeletal outputs downstream of different guidance receptors.

\section{MATERIALS AND METHODS}

\section{Contact for Reagent and Resource Sharing}

Further information and requests for resources and reagents should be directed to the Lead Contact, Greg J. Bashaw (gbashaw@pennmedicine.upenn.edu).

\section{Experimental Model and Subject Details}

\section{Genetic stocks}

The following Drosophila strains were used: $w^{1118}, \operatorname{robo}^{G A 285}, \operatorname{slit}^{2}, \operatorname{sos}^{4 G}, \operatorname{robo}^{\times 123}, \operatorname{scar}^{437}$, apGal4, egGal4, UAS-CD8GFP II, UAS-TauMycGFP III, 10XUAS-HA-Robo1 86F8, 5XUAS-HARobo1 86F8. Fly strains $h s p c 300^{454.3}$, cyfip ${ }^{485.1}$, and UAS-CYFIP were a kind gift from A. Giangrande. The genomic robo1 rescue strain robo1::HArobo1 $28 E 7$ was a kind gift from T. Evans. The following transgenic stocks were generated: 10UAS-HA-Robo 1 WIRS 86F8, 5UASHA-Robo $\triangle W I R S$ 86F8, robo1::HArobo1 1 WIRS 28E7. Transgenic flies were generated by BestGene Inc. (Chino Hills, CA) using ФC31- directed site-specific integration into landing sites at cytological position 86F8 (For UAS-Robo constructs) or 28E7 (for genomic robo1 rescue constructs). Genomic robo1::HArobo1 $\triangle$ WIRS $28 E 7$ rescue transgene was introduced onto a robo ${ }^{G A 285}$ chromosome via meiotic recombination and the presence of the robo ${ }^{G A 285}$ mutation was confirmed in all recombinant lines by DNA sequencing. The CRISPR line robo1 $\triangle W I R S$ was generated by cloning a guide targeting the WIRS motif into a pCFD3-dU6:3 backbone (Addgene, \#49410) and sending positive clones to BestGene Inc. (Chino Hills, CA) for injection. Flies were screened by PCR and restriction digest followed by DNA sequencing. All crosses were carried out at $25^{\circ} \mathrm{C}$.

\section{Mice}


Timed pregnant female CD-1 mice were obtained from Charles River. All animal work was approved by the Institutional Animal Care and Use Committee (IACUC) of the University of Pennsylvania. Embryos of both sexes were randomly used for spinal cord explants and primary dissociated neuron cultures.

\section{Dissociated Commissural Neuron Culture}

Primary commissural neuron cultures were prepared as described previously (Langlois 2010) and maintained at $5 \% \mathrm{CO}_{2}$ in a humidified incubator. Briefly, commissural neurons were isolated from E12.5 dorsal spinal cords and plated on acid-washed, Poly-D-Lysine (Sigma, \#P6407) and 2 $\mu \mathrm{g} / \mathrm{ml} \mathrm{N}$-Cadherin (R\&D, \#1388-NC) coated coverslips. Neurons were cultured in Neurobasal medium supplemented with 10\% heat-inactivated FBS (Gibco, \#10437-028) and 1X penicillin/streptomycin/glutamine (Gibco, \#10378-016). After $\sim 20 \mathrm{~h}$, the medium was replaced with Neurobasal supplemented with 1X B-27 (Thermo, \#A3582801) and the neurons were used for experiments one hour later.

\section{Explant Culture}

Dorsal spinal cord explants from E12.5 embryos were dissected and cultured in collagen gels as described previously (Serafini 1994). Briefly, explants were cultured in 50\% OptiMEM (Gibco, \#31985-070) and 45\% Ham's F-12 (Gibco, \#11765-054) media supplemented with 5\% horse-

serum (Gibco, \#16050122), $0.75 \%$ glucose (Thermo, \#D16-500) and 1X penicillin/streptomycin/glutamine for $48 \mathrm{~h}$ with 500ng/ml Netrin-1 (R\&D, \#1109-N1/CF).

\section{Cell Culture}

Drosophila S2R+ cells were maintained at $25^{\circ} \mathrm{C}$ in Schneider's media (Life Technologies, \#21720024) supplemented with 10\% (vol/vol) FBS and a mixture of $1 \%$ Penicillin and Streptomycin.

\section{Method Details}

\section{Molecular Biology}


For making the p10UAST-HA-Robo1 $\triangle W I R S$, p10UAST-Robo1 $\triangle W I R S-M Y C$ and the p5UASTHA-Robo1 $\triangle W I R S$ constructs, the wild type Robo1 coding sequences from p10UAST-HARobo1, p10UAST-Robo1-MYC and the p5UAST-HA-Robo1 constructs were subcloned into the smaller pBlueScript backbone and point mutations were introduced into the WIRS motif of the Robo coding sequences with the Quikchange II site-directed mutagenesis kit (Agilent, \#200523) using the following primers: GACACCCGTAACGCTACCGCCGCCTACGCTTGTCGCAAG and CTTGCGACAAGCGTAGGCGGTAGCGTTACGGGTGTC. The mutated Robo1 coding sequences were then subcloned back into the respective vectors with 10xUAS or 5xUAS sequences and an attB site for ФС31- directed site-specific integration. A similar strategy was used for making p10UAST-Robo2 $\triangle W I R S-M Y C$ using the following primers:

ACCGACTATGCAGAGGCGTCCGCTGCTGGCAAGGCA and

TGCCTTGCCAGCAGCGGACGCCTCTGCATAGTCGGT. For the genomic rescue robo1::HArobo1 $\triangle$ WIRS construct, the same Robo1 primers mentioned above were used to mutate the WIRS motif using Quikchange and the mutated Robo1 coding sequence was cloned into the genomic rescue construct backbone (kind gift from T. Evans) using Bg/ll.

For MYC-hRobo1 $\triangle W I R S$, the following primers were used for Quikchange:

AACAAAATCAATGAGGCGAAAGCCGCCAATAGCCCAAATCTGAAG and

CTTCAGATTTGgGCTATTGGCGgCTTTCGCCTCATTGATTTTGTT. Next, wild type MYC-hRobo1 and MYC-hRobo1 $\triangle$ WIRS coding sequences were cloned into a pCAG vector (provided by A. Kania) using Notl/Xhol sites. A signal peptide sequence was included upstream of the MYC tag. For making the p10UAST-HSPC300-GFP construct, hspc300 cDNA was PCR amplified from the pOT2 BGDP Gold Collection (clone\# Fl14118) and tagged with a C-terminal GFP separated by a linker using overlap extension PCR with the following primers:

TATATAGCGGCCGCCACCATGAGTGGGGCT and CGCGCGTCTAGATCACTTGTACAGCTCGTC and overlapping primers GGTGAAACATTAACGGGACATATGGGAGGAATGGTGAGCAAGGGC and 


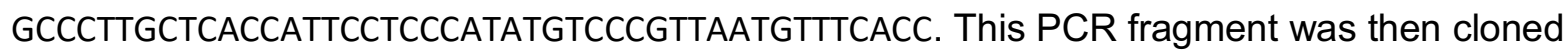
into a p10UAST plasmid containing an attB site using Notl/Xbal sites.

\section{CRISPR Cas9- mediated Mutagenesis}

For synthesizing the guide RNA to target the WIRS motif in the endogenous robo1 locus, the following sense and antisense oligonucleotides were used: GTCGGCGTACGGCGTGGGATTAT and AAACATAATCCCACGCCGTACGC. This guide RNA was selected with zero predicted off-target effects using http://targetfinder.flycrispr.neuro.brown.edu. The oligos were annealed and cloned into a Bbsl-digested pCFD3-dU6:3 vector. A single-stranded oligonucleotide template was designed to introduce point mutations into the WIRS motif. These mutations also destroy the gRNA target sequence and the PAM sequence to prevent subsequent cleavages by Cas9. An Mfel site is mutated which was used for screening potential CRISPR flies. The sequence of the template used is:

\section{CAATCCAACTACAATAACTCCGATGGAGGAACCGATTATGCAGAAGTTGACACCCGTAATGCTACCGCCGCCTACG}

CTTGTCGCAAGGTGAGGATCATATGAATTGCATCACACAACAATTTC. The template along with the pCFD3 vector containing the guide RNA was sent to BestGene Inc. (Chino Hills, CA) for injection. The progeny from these flies were crossed to balancer stocks to generate stable lines. Flies from these lines were then screened with Mfel following genomic DNA extraction and positive hits were sent for DNA sequencing.

\section{Immunoprecipitation}

S2R+ cells were transiently transfected with Effectene transfection reagent (Qiagen, Valencia CA, \#301425) and induced 24 hours later with 0.5mM copper sulfate. 24 hours after induction, cells were lysed in TBS-V (150 mM NaCl, $10 \mathrm{mM}$ Tris $\mathrm{pH}-8,1 \mathrm{mM}$ ortho-vanadate) supplemented with 0.5\% Surfact-AMPS NP40 (Thermo, Waltham MA, \#85124) and 1x Complete Protease Inhibitor (Roche, \#11697498001) for $20 \mathrm{~min}$ at $4^{\circ} \mathrm{C}$. Soluble proteins were recovered by centrifugation at 
$15,000 \times \mathrm{g}$ for $10 \mathrm{~min}$ at $4^{\circ} \mathrm{C}$. Lysates were pre-cleared with $30 \mu \mathrm{l}$ of a $50 \%$ slurry of protein $\mathrm{A}$ (Invitrogen, \#15918-014) and protein G agarose beads (Invitrogen, \#15920-010) by incubation for 20 minutes at $4{ }^{\circ} \mathrm{C}$. Pre-cleared lysates were then incubated with $0.7 \mu \mathrm{g}$ of rabbit anti-GFP antibody for 2 hours at $4^{\circ} \mathrm{C}$ to precipitate HSPC $300-$ GFP. After incubation, $30 \mu \mathrm{l}$ of a $50 \%$ slurry of protein $A$ and protein $G$ agarose beads was added and samples were incubated for an additional 30 minutes at $4^{\circ} \mathrm{C}$. The immunocomplexes were washed three times with lysis buffer, boiled for 10 min in 2x Laemmli SDS sample buffer (Bio-Rad, \#1610737) and analyzed by western blotting. Proteins were resolved by SDS-PAGE and transferred to nitrocellulose membrane (Amersham, \#10600032). Membranes were blocked with 5\% dry milk and $0.1 \%$ Tween 20 in PBS for $1 \mathrm{~h}$ at room temperature and incubated with primary antibodies overnight at $4^{\circ} \mathrm{C}$. Following three washes with PBS/0.1\% Tween 20, membranes were incubated with the appropriate HRPconjugated secondary antibody at room temperature for $1 \mathrm{~h}$. Signals were detected using Clarity ECL (Bio-Rad, \#1705061) according to manufacturer's instructions. For preparation of Slitconditioned media (Slit-CM), cells were transfected with a pUAST-Slit vector and a PMT-Gal4 vector using Effectene transfection reagent. Gal4 was induced 24 hours later with $0.5 \mathrm{mM}$ copper sulfate. 24 hours after induction, Slit-CM was collected and concentrated using Amicon filters (Amicon Ultracel 30K, Millipore, \#UFC903096). For CM treatment, cells were incubated with control-CM (prepared using an empty pUAST vector) or Slit-CM on an orbital shaker at room temperature for 12 minutes, then lysed for immunoprecipitation as described above. Antibodies used: for immunoprecipitation, rabbit anti-GFP and for western blot, rabbit anti-GFP (1:500, Invitrogen, \#a11122), mouse anti-MYC (1:1000, DSHB, \#9E10-C), mouse anti-Slit (1:50, DSHB, \#C555.6D), goat anti-rabbit HRP (1:10,000, Jackson Immunoresearch, \#111-035-003) and goat anti-mouse HRP (1:10,000, Jackson Immunoresearch, \#115-035-146).

For co-immunoprecipitation assays in Drosophila embryos, embryonic protein lysates were prepared from approximately $100 \mu \mathrm{l}$ of embryos overexpressing UAS-HSPC300-GFP alone or with the HA-tagged UAS-Robo1 variants in all neurons. Embryos were lysed in $0.5 \mathrm{ml}$ TBS-V (150 
$\mathrm{mM} \mathrm{NaCl}, 10 \mathrm{mM}$ Tris $\mathrm{pH} 8.0,1 \mathrm{mM}$ ortho-vanadate) supplemented with 1\% Surfact-AMPS NP40 and protease inhibitors by manual homogenization using a plastic pestle. Homogenized samples were incubated with gentle rocking at $4^{\circ} \mathrm{C}$ for $10 \mathrm{~min}$ and centrifuged at $15,000 \mathrm{xg}$ for $10 \mathrm{~min}$ in a pre-chilled rotor. Supernatants were collected after centrifugation and immunoprecipitations and western blotting were performed as described above. Antibodies used: for immunoprecipitation, rabbit anti-GFP (1:500, Invitrogen, \#a11122) and for western blot, rabbit anti-GFP (1:500, Invitrogen, \#a11122), mouse anti-HA (1:1000, BioLegend, \#901502), mouse anti-beta tubulin (1:1000, DSHB, \#E7), goat anti-rabbit HRP (1:10,000, Jackson Immunoresearch, \#111-035-003) and goat anti-mouse HRP (1:10,000, Jackson Immunoresearch, \#115-035-146).

\section{Immunostaining}

Dechorionated, formaldehyde-fixed Drosophila embryos were fluorescently stained using standard methods. The following antibodies were used: rabbit anti-GFP (1:250, Invitrogen, \#a11122), mouse anti-HA (1:500, BioLegend, \#901502), chick anti-beta gal (1:500, Abcam, \#ab9361), mouse anti-Scar (1:50, DSHB, \#P1C1), mouse anti-Robo (1:50, DSHB, \#13C9), Alexa647 goat anti-HRP (1:500, Jackson Immunoresearch, \#123-605-021), Alexa488 goat antirabbit (1:500, Invitrogen, \#A11034), Alexa488 goat anti-mouse (1:500, Invitrogen, \#A11029), Alexa488 goat anti-chick (1:500, Invitrogen, \#A11039), Cy3 goat anti-mouse (1:500, Jackson Immunoresearch, \#115-165-003), Cy3 goat anti-Chick (1:500, Abcam, \#ab97145) and goat antimouse HRP (1:10,000, Jackson Immunoresearch, \#115-035-146). Embryos were filleted and mounted in $70 \%$ glycerol/1xPBS.

Dissociated spinal commissural neurons were fixed for 20 min in 4\% paraformaldehyde (Electron Microscopy Services, \#15710) at room temperature and washed three times with PBS. Fixed neurons were then permeabilized with $0.1 \%$ Triton X-100 in PBS (PBT) for 10 min and blocked with $2 \%$ horse serum (HS) in PBT for $30 \mathrm{~min}$ at room temperature. The blocking solution was replaced with primary antibody diluted in $2 \% \mathrm{HS}$ in PBT and incubated overnight at $4^{\circ} \mathrm{C}$. Following three washes with PBT, secondary antibody diluted in $2 \%$ HS in PBT was added and incubated 
for $1 \mathrm{~h}$ at room temperature. Neurons were then washed three times with PBT and the coverslips were mounted in Aquamount. The following antibodies were used: mouse anti-MYC (1:500, DSHB, \#9E10-C), goat anti-Robo3 (1:200, R\&D systems, \#AF3076), Cy3 donkey anti-goat (1:500, Jackson Immunoresearch, \#705-165-147) and 488 donkey anti-goat (1:500, Jackson Immunoresearch, \#715-545-150).

Collagen-embedded explants were fixed in $4 \%$ paraformaldehyde overnight at $4{ }^{\circ} \mathrm{C}$ and washed three times for $10 \mathrm{~min}$ in PBS. Fixed explants were then blocked in $2.5 \%$ normal goat serum (NGS) in PBT for $2 \mathrm{~h}$ at room temperature and incubated with primary antibody diluted in blocking solution overnight at $4^{\circ} \mathrm{C}$. Explants were washed six times for $1 \mathrm{~h}$ with PBT and incubated with secondary antibody diluted in blocking solution overnight at $4^{\circ} \mathrm{C}$. After $61 \mathrm{~h}$ washes with PBT, explants were mounted on cavity slides using Aquamount. The following antibodies were used: mouse anti-MYC (1:500, DSHB, \#9E10-C), mouse anti-beta tubulin (1:300, DSHB, \#E7), rabbit anti-dsRed (1:200, Takara, \#632496), Alexa488 goat anti-mouse (1:500, Invitrogen, \#A11029) and Cy3 goat anti-rabbit (1:500, Jackson Immunoresearch, \#111-165-003).

Fixed samples of Drosophila embryo nerve cords, mouse dissociated commissural neurons and mouse dorsal spinal cord explants were imaged using a spinning disk confocal system (Perkin Elmer) built on a Nikon Ti-U inverted microscope using a Nikon 40X objective (for nerve cords and neurons) and a 10X objective (for explants) with Volocity imaging software. Images were processed using NIH ImageJ software.

\section{Electroporation of Mouse Embryos and Primary Neuron Culture}

E12.5 embryos were electroporated ex utero by injecting $100 \mathrm{ng} / \mu \mathrm{l}$ DNA in electroporation buffer (30mM HEPES pH7.5 (Thermo, \#BP299-1), 300mM KCl (Thermo, \#BP366-1), 1mM MgCl 2 (Thermo, \#BP214-500) and 0.1\% Fast Green FCF (Thermo, \#F99-10)) into the central canal of the neural tube. A BTX ECM 830 electroporator (BTX Harvard Apparatus, \#45-0662) was used for bilateral electroporation into spinal cord neurons (Five $30 \mathrm{~V}$ pulses, each of $50 \mathrm{~ms}$ duration for each half of the spinal cord). Following electroporation, dorsal spinal cords were dissected out 
and cut into explants for the explant outgrowth assay or used for preparation of dissociated neuronal cultures. For neuron culture, dissected spinal cords were washed in Hanks' Balanced Salt Solution (HBSS, Gibco, \#14175-079) and digested with 0.05\% trypsin (Gibco, \#25300054) for $7 \mathrm{~min}$ at $37^{\circ} \mathrm{C}$. $1 \mu \mathrm{l}$ of DNase I (NEB, \#M0303L) and $0.15 \% \mathrm{MgSO}_{4}$ (Thermo, \#7487-88-9) was added for an additional minute and the samples were centrifuged at $400 \times \mathrm{g}$ for $4 \mathrm{~min}$. Samples were washed with pre-warmed HBSS and a small fire-polished Pasteur pipette was used to triturate the tissue and dissociate it into single cells. Cells were plated on acid-washed, Poly-DLysine and N-Cadherin coated coverslips and cultured in plating media (Neurobasal (Gibco, \#21103-049) medium supplemented with 10\% heat-inactivated FBS and 1X penicillin/streptomycin/glutamine).

\section{Explant Outgrowth Assay}

Dorsal spinal cord explants form E12.5 mouse embryos were dissected and cultured in collagen gels as previously described (Serafini 1994). Briefly, explants were embedded in rat tail collagen (Corning, \#354249) gels at a distance of one explant diameters away from a mock 293T cell aggregate (ATCC, CRL-3216) or a cell aggregate expressing Slit (pSecTagB-hSlit2-MYC, kind gift from A. Chedotal). Explants were grown in 50\% OptiMEM and 45\% Ham's F-12 media supplemented with $5 \%$ horse-serum, $0.75 \%$ glucose and $1 \mathrm{X}$ penicillin/streptomycin/glutamine for $48 \mathrm{~h}$ with $500 \mathrm{ng} / \mathrm{ml}$ Netrin-1. Explant were subsequently fixed and stained as described above. For preparation of $293 \mathrm{~T}$ cell aggregates, cells were trypsinized and resuspended in a rat tail collagen solution, drawn into a glass Pasteur pipette and allowed to polymerize. Release the collagen-embedded cells from the pipette using a rubber bulb and cut the aggregates into $1 \mathrm{~mm}$ clusters.

\section{Collapse Assay}

Dissociated commissural neurons from E12.5 mouse embryos were cultured in plating media (Neurobasal medium supplemented with 10\% heat-inactivated FBS and 1X penicillin/streptomycin/glutamine) for 1 day in vitro. Plating media was replaced with Neurobasal 
supplemented with 1 X B-27 for 1 hour. Neurons were treated with recombinant hSlit2-N (R\&D, \#5444-SL-050) at $2 \mu \mathrm{g} / \mathrm{ml}$ for 30 mins at $37^{\circ} \mathrm{C}$. Neurons were fixed immediately and immunostained for Robo3 (a marker for commissural neurons) and MYC to identify neurons that had been successfully electroporated with the hRobo1-MYC or hRobo1 $\Delta$ WIRS-MYC expression constructs.

\section{Quantification and Statistical Analysis}

For analysis of Drosophila nerve cord phenotypes, image analysis was conducted blind to the genotype. Data are presented as mean values \pm S.E.M. For statistical analysis, comparisons were made between two groups using the Student's $t$-test. For multiple comparisons, significance was assessed using one-way ANOVA with Tukey's post hoc tests. Differences were considered significant when $p<0.05$. For the collapse assay, only Robo3-positive (and MYC-positive for neurons electroporated with hRobo1 variants) axons were imaged and analyzed. Growth cones were defined by the presence of lamellipodia and/or filopodia. 3 trials were conducted and at least 30 neurons per condition were scored in each trial. Data are presented as mean values \pm S.E.M. For statistical analysis, comparisons were made between groups using one-way ANOVA with Tukey's post hoc tests. Differences were considered significant when $p<0.05$. For the explant outgrowth assay, explants images were converted to black-and-white composites using the Threshold function. Each experimental set was quantified using the same threshold parameters. Explant quadrants were delineated by placing a right-angled crosshair at the center of each explant with the proximal quadrant directly facing the cell aggregate. The total area of black pixels was measured for the proximal and distal quadrants using the Analyze Particles function. The particles showing axonal outgrowth were then erased using the Eraser tool and the total area of black particles was measured again. The difference was recorded as total area of axonal outgrowth. Next, the length of each quadrant was measured by tracing the border of the quadrant using the Freehand Line tool. Values for total area of outgrowth were normalized to length of the 
quadrant and these final values were used to obtain the proximal/distal ratios for each explant. The measurements for each explant in a set were averaged and the ratios of experimental conditions compared with control condition were calculated. Data are presented as mean \pm SEM. Total number of explants for RFP control, RFP Slit, hRobo1 control, hRobo1 Slit, hRobo1 $\Delta$ WIRS control and hRobo1 $\Delta$ WIRS Slit is 29, 39, 33, 39, 29 and 41 respectively (from 3 independent experiments). For statistical analysis, comparisons were made between groups using one-way ANOVA with Tukey's post hoc tests. Differences were considered significant when $p<0.05$. For western blots, densitometric analysis was performed and band intensities of co-immunoprecipitating proteins in the immunoprecipitates were normalized to band intensities of HSPC300 in the immunoprecipitates as well as to lysate levels of the co-immunoprecipitating proteins. For each independent experiment, values were compared with wild type Robo1 normalized values. Data are presented as mean \pm SEM. For statistical analysis, comparisons were made between two groups using the Student's $t$-test. For multiple comparisons, significance was assessed using one-way ANOVA with Tukey's post hoc tests. Differences were considered significant when $p<0.05$.

\section{Data and Software Availability:}

Confocal stacks were collected using a spinning disk confocal system (Perkin Elmer) built on a Nikon Ti-U inverted microscope with Volocity imaging software. Images were processed using $\mathrm{NIH}$ ImageJ and Adobe Photoshop software. All statistics and graphs were generated using GraphPad Prism 8.

\section{Author Contributions}

K.C. designed and performed all of the genetic and biochemical experiments and wrote the paper. M.G. and K.C. designed and performed the in vitro axon guidance assays in mouse dorsal commissural axons. A.K. and C.C. designed and performed experiments. K.C., M.G., 
C.C analyzed the data, G.J.B. designed the experiments, wrote the paper and supervised the project.

\section{Acknowledgments}

We would like to acknowledge members of the Bashaw Lab for discussions and comments on the manuscript. We thank Zachary DeLoughery and Alexander Jaworski for guidance on the in vitro explant experiments and Timothy Evans for the gift of the genomic Robo rescue construct. NSF Grant IOS-1853719 and NIH Grant R35 NS097340 to G.J.B supported this research. 


\section{REFERENCES}

Abekhoukh, S., Bardoni, B., 2014. CYFIP family proteins between autism and intellectual disability: links with Fragile $X$ syndrome. Front Cell Neurosci 8, 81.

Anitha, A., Nakamura, K., Yamada, K., Suda, S., Thanseem, I., Tsujii, M., Iwayama, Y., Hattori, E., Toyota, T., Miyachi, T., Iwata, Y., Suzuki, K., Matsuzaki, H., Kawai, M., Sekine, Y., Tsuchiya, K., Sugihara, G., Ouchi, Y., Sugiyama, T., Koizumi, K., Higashida, H., Takei, N., Yoshikawa, T., Mori, N., 2008. Genetic analyses of roundabout (ROBO) axon guidance receptors in autism. 147B, 1019-1027.

Ballard, M.S., Zhu, A., Iwai, N., Stensrud, M., Mapps, A., Postiglione, M.P., Knoblich, J.A., Hinck, L., 2015. Mammary Stem Cell Self-Renewal Is Regulated by Slit2/Robo1 Signaling through SNAI1 and mINSC. Cell Rep 13, 290-301.

Barzik, M., Kotova, T.I., Higgs, H.N., Hazelwood, L., Hanein, D., Gertler, F.B., Schafer, D.A., 2005. Ena/VASP proteins enhance actin polymerization in the presence of barbed end capping proteins. J Biol Chem 280, 28653-28662.

Bashaw, G.J., Kidd, T., Murray, D., Pawson, T., Goodman, C.S., 2000. Repulsive axon guidance: Abelson and Enabled play opposing roles downstream of the roundabout receptor. Cell 101, 703-715.

Basquin, C., Trichet, M., Vihinen, H., Malarde, V., Lagache, T., Ripoll, L., Jokitalo, E., Olivo-Marin, J.C., Gautreau, A., Sauvonnet, N., 2015. Membrane protrusion powers clathrin-independent endocytosis of interleukin-2 receptor. EMBO J 34, 2147-2161.

Battye, R., Stevens, A., Jacobs, J.R., 1999. Axon repulsion from the midline of the Drosophila CNS requires slit function. Development 126, 2475-2481.

Bogdan, S., Stephan, R., Lobke, C., Mertens, A., Klambt, C., 2005. Abi activates WASP to promote sensory organ development. Nat Cell Biol 7, 977-984.

Bosley, T.M., Salih, M.A., Jen, J.C., Lin, D.D., Oystreck, D., Abu-Amero, K.K., MacDonald, D.B., al Zayed, Z., al Dhalaan, H., Kansu, T., Stigsby, B., Baloh, R.W., 2005. Neurologic features of horizontal gaze palsy and progressive scoliosis with mutations in ROBO3. Neurology 64, 11961203.

Brose, K., Bland, K.S., Wang, K.H., Arnott, D., Henzel, W., Goodman, C.S., Tessier-Lavigne, M., Kidd, T., 1999. Slit proteins bind Robo receptors and have an evolutionarily conserved role in repulsive axon guidance. Cell 96, 795-806.

Brown, H.E., Reichert, M.C., Evans, T.A., 2015. Slit Binding via the Ig1 Domain Is Essential for Midline Repulsion by Drosophila Robo1 but Dispensable for Receptor Expression, Localization, and Regulation in Vivo. G3 (Bethesda) 5, 2429-2439.

Chance, R.K., Bashaw, G.J., 2015. Slit-Dependent Endocytic Trafficking of the Robo Receptor Is Required for Son of Sevenless Recruitment and Midline Axon Repulsion. PLoS Genet 11, e1005402.

Chen, B., Brinkmann, K., Chen, Z., Pak, C.W., Liao, Y., Shi, S., Henry, L., Grishin, N.V., Bogdan, S., Rosen, M.K., 2014a. The WAVE regulatory complex links diverse receptors to the actin cytoskeleton. Cell 156, 195-207.

Chen, X.J., Squarr, A.J., Stephan, R., Chen, B., Higgins, T.E., Barry, D.J., Martin, M.C., Rosen, M.K., Bogdan, S., Way, M., 2014b. Ena/VASP proteins cooperate with the WAVE complex to regulate the actin cytoskeleton. Dev Cell 30, 569-584. 
Chen, Z., Borek, D., Padrick, S.B., Gomez, T.S., Metlagel, Z., Ismail, A.M., Umetani, J., Billadeau, D.D., Otwinowski, Z., Rosen, M.K., 2010. Structure and control of the actin regulatory WAVE complex. Nature 468, 533-538.

Chia, P.H., Chen, B., Li, P., Rosen, M.K., Shen, K., 2014. Local F-actin network links synapse formation and axon branching. Cell 156, 208-220.

Coleman, H.A., Labrador, J.P., Chance, R.K., Bashaw, G.J., 2010. The Adam family

metalloprotease Kuzbanian regulates the cleavage of the roundabout receptor to control axon repulsion at the midline. Development 137, 2417-2426.

Depienne, C., Cincotta, M., Billot, S., Bouteiller, D., Groppa, S., Brochard, V., Flamand, C., Hubsch, C., Meunier, S., Giovannelli, F., Klebe, S., Corvol, J.C., Vidailhet, M., Brice, A., Roze, E., 2011. A novel DCC mutation and genetic heterogeneity in congenital mirror movements. Neurology 76, 260-264.

Eden, S., Rohatgi, R., Podtelejnikov, A.V., Mann, M., Kirschner, M.W., 2002. Mechanism of regulation of WAVE1-induced actin nucleation by Rac1 and Nck. Nature 418, 790-793.

Englund, C., Steneberg, P., Falileeva, L., Xylourgidis, N., Samakovlis, C., 2002. Attractive and repulsive functions of Slit are mediated by different receptors in the Drosophila trachea.

Development 129, 4941-4951.

Evans, T.A., Bashaw, G.J., 2010. Functional diversity of Robo receptor immunoglobulin domains promotes distinct axon guidance decisions. Curr Biol 20, 567-572.

Fan, X., Labrador, J.P., Hing, H., Bashaw, G.J., 2003. Slit stimulation recruits Dock and Pak to the roundabout receptor and increases Rac activity to regulate axon repulsion at the CNS midline.

Neuron 40, 113-127.

Forsthoefel, D.J., Liebl, E.C., Kolodziej, P.A., Seeger, M.A., 2005. The Abelson tyrosine kinase, the Trio GEF and Enabled interact with the Netrin receptor Frazzled in Drosophila. Development 132, 1983-1994.

Gitai, Z., Yu, T.W., Lundquist, E.A., Tessier-Lavigne, M., Bargmann, C.I., 2003. The netrin receptor UNC-40/DCC stimulates axon attraction and outgrowth through enabled and, in parallel, Rac and UNC-115/AbLIM. Neuron 37, 53-65.

Hannula-Jouppi, K., Kaminen-Ahola, N., Taipale, M., Eklund, R., Nopola-Hemmi, J., Kaariainen, $\mathrm{H} .$, Kere, J., 2005. The axon guidance receptor gene ROBO1 is a candidate gene for developmental dyslexia. PLoS Genet 1, e50.

Hsouna, A., Kim, Y.S., VanBerkum, M.F., 2003. Abelson tyrosine kinase is required to transduce midline repulsive cues. J Neurobiol 57, 15-30.

Ismail, A.M., Padrick, S.B., Chen, B., Umetani, J., Rosen, M.K., 2009. The WAVE regulatory complex is inhibited. Nat Struct Mol Biol 16, 561-563.

Jaworski, A., Tom, I., Tong, R.K., Gildea, H.K., Koch, A.W., Gonzalez, L.C., Tessier-Lavigne, M., 2015. Operational redundancy in axon guidance through the multifunctional receptor Robo3 and its ligand NELL2. Science 350, 961-965.

Jen, J.C., Chan, W.M., Bosley, T.M., Wan, J., Carr, J.R., Rub, U., Shattuck, D., Salamon, G., Kudo, L.C., Ou, J., Lin, D.D., Salih, M.A., Kansu, T., Al Dhalaan, H., Al Zayed, Z., MacDonald, D.B., Stigsby, B., Plaitakis, A., Dretakis, E.K., Gottlob, I., Pieh, C., Traboulsi, E.I., Wang, Q., Wang, L., Andrews, C., Yamada, K., Demer, J.L., Karim, S., Alger, J.R., Geschwind, D.H., Deller, T., Sicotte, N.L., Nelson, S.F., Baloh, R.W., Engle, E.C., 2004. Mutations in a human ROBO gene disrupt hindbrain axon pathway crossing and morphogenesis. Science 304, 1509-1513. 
Kidd, T., Bland, K.S., Goodman, C.S., 1999. Slit is the midline repellent for the robo receptor in Drosophila. Cell 96, 785-794.

Kidd, T., Brose, K., Mitchell, K.J., Fetter, R.D., Tessier-Lavigne, M., Goodman, C.S., Tear, G., 1998. Roundabout controls axon crossing of the CNS midline and defines a novel subfamily of evolutionarily conserved guidance receptors. Cell 92, 205-215.

Kinoshita-Kawada, M., Hasegawa, H., Hongu, T., Yanagi, S., Kanaho, Y., Masai, I., Mishima, T., Chen, X., Tsuboi, Y., Rao, Y., Yuasa-Kawada, J., Wu, J.Y., 2019. A crucial role for Arf6 in the response of commissural axons to Slit. Development 146.

Kitamura, T., Kitamura, Y., Yonezawa, K., Totty, N.F., Gout, I., Hara, K., Waterfield, M.D., Sakaue, M., Ogawa, W., Kasuga, M., 1996. Molecular cloning of p125Nap1, a protein that associates with an SH3 domain of Nck. Biochem Biophys Res Commun 219, 509-514.

Klambt, C., Jacobs, J.R., Goodman, C.S., 1991. The midline of the Drosophila central nervous system: a model for the genetic analysis of cell fate, cell migration, and growth cone guidance. Cell 64, 801-815.

Koohini, Z., Koohini, Z., Teimourian, S., 2019. Slit/Robo Signaling Pathway in Cancer; a New Stand Point for Cancer Treatment. Pathol Oncol Res 25, 1285-1293.

Kramer, S.G., Kidd, T., Simpson, J.H., Goodman, C.S., 2001. Switching repulsion to attraction: changing responses to slit during transition in mesoderm migration. Science 292, 737-740. Kraut, R., Zinn, K., 2004. Roundabout 2 regulates migration of sensory neurons by signaling in trans. Curr Biol 14, 1319-1329.

Lee, N.K., Fok, K.W., White, A., Wilson, N.H., O'Leary, C.J., Cox, H.L., Michael, M., Yap, A.S., Cooper, H.M., 2016. Neogenin recruitment of the WAVE regulatory complex maintains adherens junction stability and tension. Nat Commun 7, 11082.

Leng, Y., Zhang, J., Badour, K., Arpaia, E., Freeman, S., Cheung, P., Siu, M., Siminovitch, K., 2005. Abelson-interactor-1 promotes WAVE2 membrane translocation and Abelson-mediated tyrosine phosphorylation required for WAVE2 activation. Proc Natl Acad Sci U S A 102, 10981103.

Macias, H., Moran, A., Samara, Y., Moreno, M., Compton, J.E., Harburg, G., Strickland, P., Hinck, L., 2011. SLIT/ROBO1 signaling suppresses mammary branching morphogenesis by limiting basal cell number. Dev Cell 20, 827-840.

McConnell, R.E., Edward van Veen, J., Vidaki, M., Kwiatkowski, A.V., Meyer, A.S., Gertler, F.B., 2016. A requirement for filopodia extension toward Slit during Robo-mediated axon repulsion. J Cell Biol 213, 261-274.

Mommersteeg, M.T., Andrews, W.D., Ypsilanti, A.R., Zelina, P., Yeh, M.L., Norden, J., Kispert, A., Chedotal, A., Christoffels, V.M., Parnavelas, J.G., 2013. Slit-roundabout signaling regulates the development of the cardiac systemic venous return and pericardium. Circ Res 112, 465-475. Qian, L., Liu, J., Bodmer, R., 2005. Slit and Robo control cardiac cell polarity and morphogenesis. Curr Biol 15, 2271-2278.

Rajagopalan, S., Vivancos, V., Nicolas, E., Dickson, B.J., 2000. Selecting a longitudinal pathway: Robo receptors specify the lateral position of axons in the Drosophila CNS. Cell 103, 1033-1045. Rama, N., Dubrac, A., Mathivet, T., Ni Charthaigh, R.A., Genet, G., Cristofaro, B., PibouinFragner, L., Ma, L., Eichmann, A., Chedotal, A., 2015. Slit2 signaling through Robo1 and Robo 2 is required for retinal neovascularization. Nat Med 21, 483-491. 
Ridley, A.J., Paterson, H.F., Johnston, C.L., Diekmann, D., Hall, A., 1992. The small GTP-binding protein rac regulates growth factor-induced membrane ruffling. Cell 70, 401-410. Ryu, J.R., Echarri, A., Li, R., Pendergast, A.M., 2009. Regulation of cell-cell adhesion by Abi/Diaphanous complexes. Mol Cell Biol 29, 1735-1748.

Schenck, A., Bardoni, B., Langmann, C., Harden, N., Mandel, J.L., Giangrande, A., 2003. CYFIP/Sra-1 controls neuronal connectivity in Drosophila and links the Rac1 GTPase pathway to the fragile $X$ protein. Neuron 38, 887-898.

Schenck, A., Bardoni, B., Moro, A., Bagni, C., Mandel, J.L., 2001. A highly conserved protein family interacting with the fragile $X$ mental retardation protein (FMRP) and displaying selective interactions with FMRP-related proteins FXR1P and FXR2P. Proc Natl Acad Sci U S A 98, 88448849.

Schenck, A., Qurashi, A., Carrera, P., Bardoni, B., Diebold, C., Schejter, E., Mandel, J.L., Giangrande, A., 2004. WAVE/SCAR, a multifunctional complex coordinating different aspects of neuronal connectivity. Dev Biol 274, 260-270.

Seeger, M., Tear, G., Ferres-Marco, D., Goodman, C.S., 1993. Mutations affecting growth cone guidance in Drosophila: genes necessary for guidance toward or away from the midline. Neuron 10, 409-426.

Shakir, M.A., Jiang, K., Struckhoff, E.C., Demarco, R.S., Patel, F.B., Soto, M.C., Lundquist, E.A., 2008. The Arp2/3 activators WAVE and WASP have distinct genetic interactions with Rac GTPases in Caenorhabditis elegans axon guidance. Genetics 179, 1957-1971.

Simpson, J.H., Bland, K.S., Fetter, R.D., Goodman, C.S., 2000. Short-range and long-range guidance by Slit and its Robo receptors: a combinatorial code of Robo receptors controls lateral position. Cell 103, 1019-1032.

Spitzweck, B., Brankatschk, M., Dickson, B.J., 2010. Distinct protein domains and expression patterns confer divergent axon guidance functions for Drosophila Robo receptors. Cell 140, 409-420.

Stephan, R., Gohl, C., Fleige, A., Klambt, C., Bogdan, S., 2011. Membrane-targeted WAVE mediates photoreceptor axon targeting in the absence of the WAVE complex in Drosophila. Mol Biol Cell 22, 4079-4092.

Suda, S., Iwata, K., Shimmura, C., Kameno, Y., Anitha, A., Thanseem, I., Nakamura, K., Matsuzaki, H., Tsuchiya, K.J., Sugihara, G., Iwata, Y., Suzuki, K., Koizumi, K., Higashida, H., Takei, N., Mori, N., 2011. Decreased expression of axon-guidance receptors in the anterior cingulate cortex in autism. Mol Autism 2, 14.

Tong, M., Jun, T., Nie, Y., Hao, J., Fan, D., 2019. The Role of the Slit/Robo Signaling Pathway. J Cancer 10, 2694-2705.

Wong, K., Ren, X.R., Huang, Y.Z., Xie, Y., Liu, G., Saito, H., Tang, H., Wen, L., Brady-Kalnay, S.M., Mei, L., Wu, J.Y., Xiong, W.C., Rao, Y., 2001. Signal transduction in neuronal migration: roles of GTPase activating proteins and the small GTPase Cdc42 in the Slit-Robo pathway. Cell 107, 209221.

Xing, G., Li, M., Sun, Y., Rui, M., Zhuang, Y., Lv, H., Han, J., Jia, Z., Xie, W., 2018. NeurexinNeuroligin 1 regulates synaptic morphology and functions via the WAVE regulatory complex in Drosophila neuromuscular junction. Elife 7.

$\mathrm{Xu}, \mathrm{C} ., \mathrm{Fu}, \mathrm{X} ., \mathrm{Zhu}, \mathrm{S}$., Liu, J.J., 2016. Retrolinkin recruits the WAVE1 protein complex to facilitate BDNF-induced TrkB endocytosis and dendrite outgrowth. Mol Biol Cell 27, 3342-3356. 
Xu, Y., Quinn, C.C., 2012. MIG-10 functions with ABI-1 to mediate the UNC-6 and SLT-1 axon guidance signaling pathways. PLoS Genet 8, e1003054.

Yang, C., Svitkina, T., 2011. Filopodia initiation: focus on the Arp2/3 complex and formins. Cell Adh Migr 5, 402-408.

Yang, L., Bashaw, G.J., 2006. Son of sevenless directly links the Robo receptor to rac activation to control axon repulsion at the midline. Neuron 52, 595-607.

Zallen, J.A., Cohen, Y., Hudson, A.M., Cooley, L., Wieschaus, E., Schejter, E.D., 2002. SCAR is a primary regulator of Arp2/3-dependent morphological events in Drosophila. J Cell Biol 156, 689701. 


\section{SUPPLEMENTARY INFORMATION}

Inventory of Supplemental Information

Figure S1 and legend: Scar expression in wild type and scar mutant embryos.

(Related to Figure 1)

Figure S2 and legend: Robo2 interaction with the WRC is entirely dependent on its WIRS motif. (Related to Figures 2 and 3)

Figure S3 and legend: 10XUAS-Robo1 rescue of the robo mutant phenotype.

(Related to Figure 4)

Figure S4: Comparable expression of the genomic rescue transgenes.

(Related to Figure 5)

Figure S5 and legend: Schematic for CRISPR-Cas9 mutagenesis and Robo1 staining in CRIPR robo $\Delta$ WIRS embryos. (Related to Figure 5)

Figure S6 and legend: Expression of hRobo1 variants electroporated into dorsal spinal commissural neurons. (Related to Figure 6) 
FIGURE S1

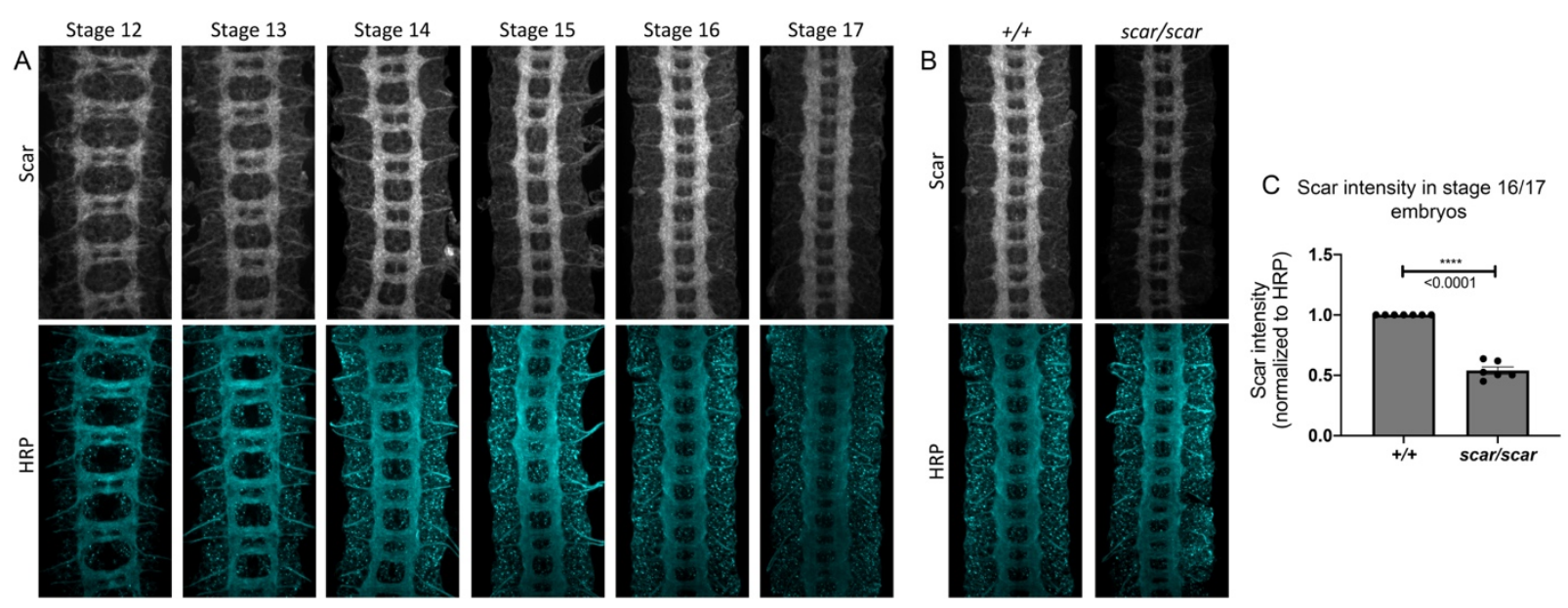

Supplementary figure 1. Scar expression in wild type and scar mutant embryos. (A) Wild type embryos across developmental stages 12 to 17 stained with anti-Scar and anti-HRP show Scar enrichment in developing CNS axons. (B) Stage 16 wild type and scar mutant embryos stained with anti-Scar and anti-HRP. Scar protein levels are reduced in scar mutant embryos. (C) Quantification of relative fluorescence intensity of Scar in CNS axons of stage 16/17 embryos calculated as Scar fluorescence intensity divided by HRP fluorescence intensity. Data are presented as mean \pm SEM, number of embryos, $n=7$, 6 . Significance was assessed using Student's $t$-test. 
FIGURE S2

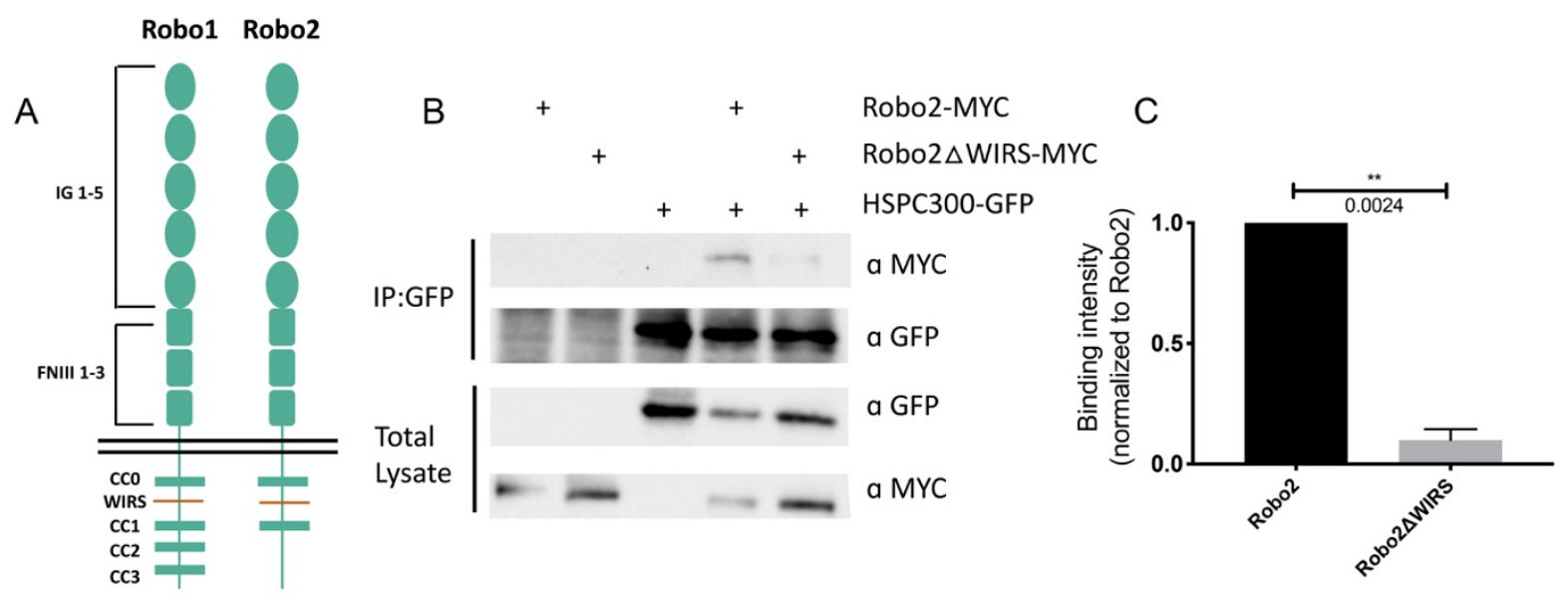

Supplementary figure 2. Robo2 interaction with the WRC is entirely dependent on its WIRS motif. (A) Schematic of Drosophila Robo1 and Robo2 receptors. Both Robo1 and Robo2 have a WIRS motif between CCO and CC1 but Robo2 lacks the CC2 and CC3 motifs that are present in Robo1. (B) Drosophila S2R+ cell lysates co-expressing HSPC300-GFP with either wild type Robo2-MYC or Robo2 $\triangle$ WIRS-MYC were immunoprecipitated with an anti-GFP antibody. The first three lanes show the individual proteins expressed alone. The fourth lane shows wild type Robo2 co-immunoprecipitating with HSPC300 while the fifth lane shows that mutating the WIRS motif completely abolishes this binding. (C) Quantitative representations of band intensities of the MYC-tagged Robo2 variants in the immunoprecipitates normalized to wild type Robo2-MYC. Data were normalized to lysate levels of the Robo2 variants and HSPC300 levels in the immunoprecipitates. Error bars represent SEM. Number of trials, $n=2$. Significance was assessed using Student's t-test. 
FIGURE S3

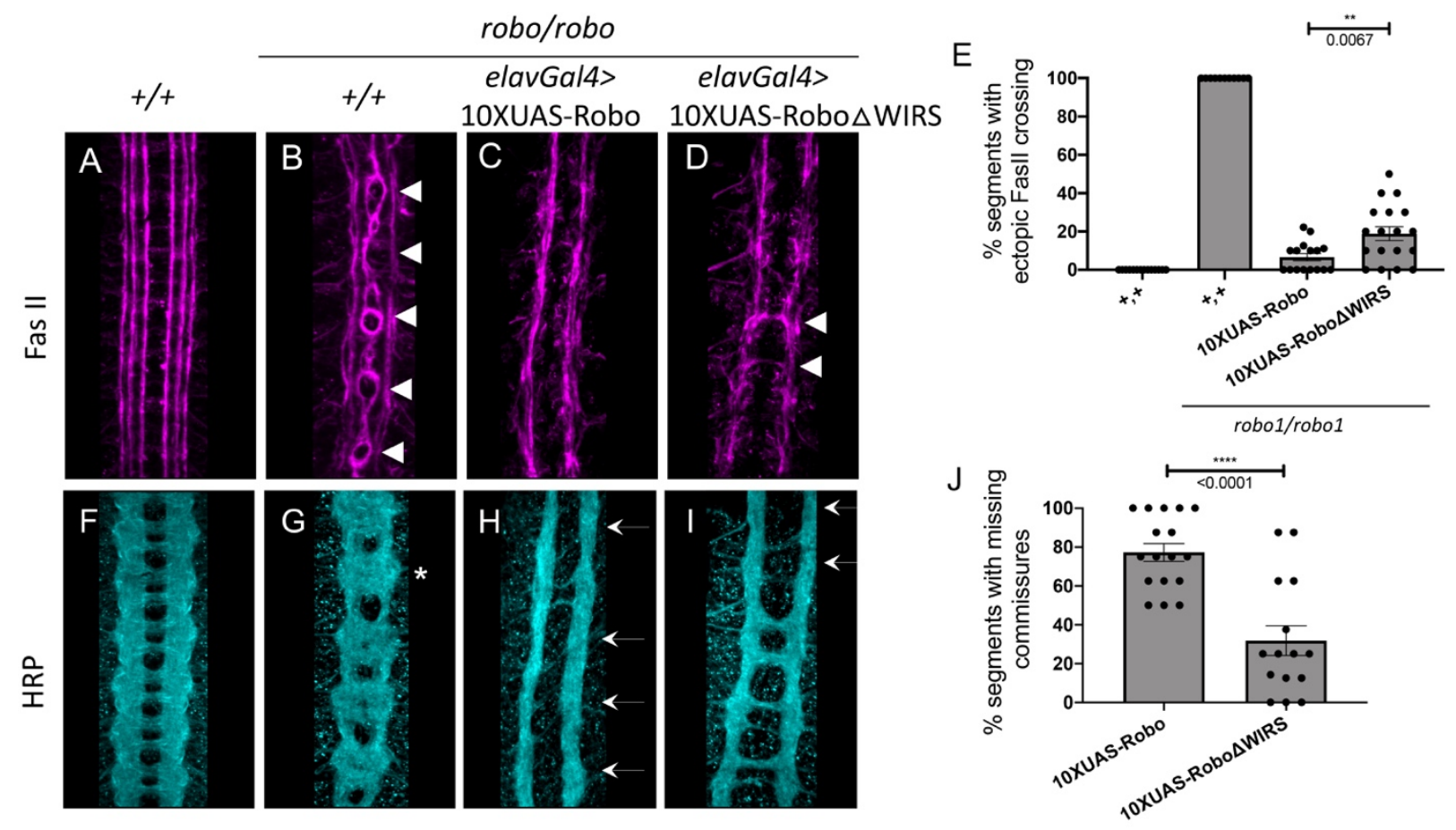

Supplementary figure 3. 10XUAS-Robo1 rescue of the robo mutant phenotype. (A-D) Stage 17 embryos stained with anti-Fasll. (A) Wild type embryos show no phenotype in Fasll. (B) robo mutants show severe ectopic Fasll crossing defects in $100 \%$ of segments (arrowheads). (C) Panneuronal expression of wild type 10XUAS-Robo1 completely rescues the robo mutant phenotype in Fasll. (D) Pan-neuronal expression of 10XUAS-Robo1 1 WIRS results in a mildly weaker rescue with a small number of Fasll bundles still crossing the midline. (E) Quantitation shows the percentage of segments in which Fasll axons ectopically cross the midline. Data are presented as mean $\pm S E M$, number of embryos, $n=14,11,16,18$. Significance was assessed using Student's $t$-test. (F-I) Stage 17 embryos stained with anti-HRP. (F) Wild type embryos show no phenotype in HRP. (G) robo mutants show a strong HRP phenotype with thickening and fusion of commissures (asterisk). (H) Pan-neuronal expression of wild type 10XUAS-Robo1 gives the opposite phenotype with strong ectopic repulsion of commissural axons resulting in segments with a complete absence of commissures (arrows). (I) In contrast, pan-neuronal expression of 10XUAS-Robo1 UWIRS shows a significantly reduced ability to induce ectopic repulsion in commissural axons resulting in much fewer segments with missing commissures. (J) Quantitation shows the percentage of segments with missing commissures. Data are presented as mean $\pm S E M$, number of embryos, $n=16,18$. Significance was assessed using Student's $t$ test. 


\section{FIGURE S4}

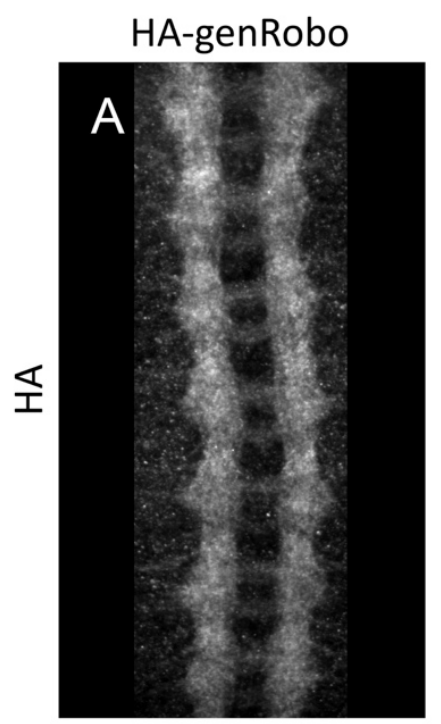

\section{HA-genRobo $\triangle$ WIRS}
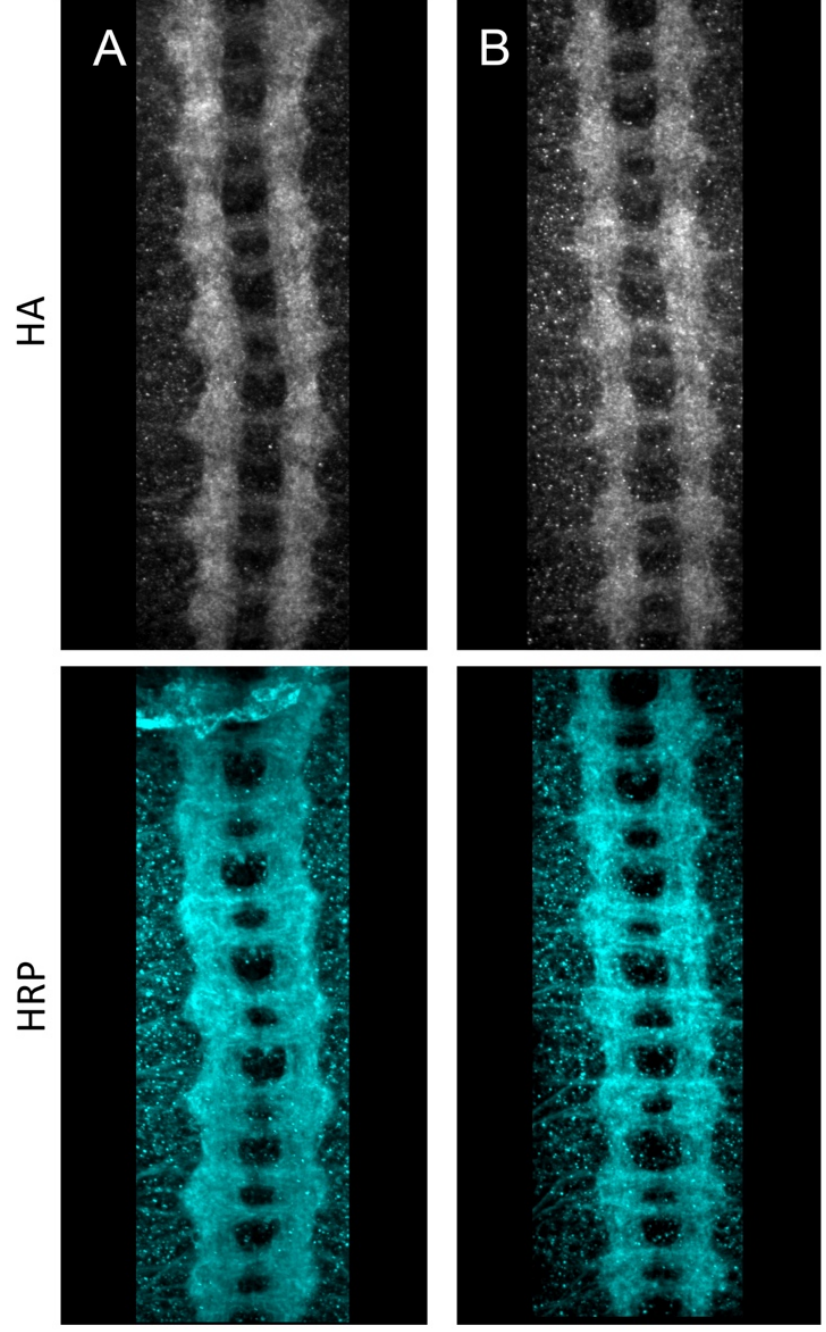

Supplementary figure 4. Comparable expression of the genomic rescue transgenes. (A) Stage 17 embryo expressing the genomic HA-tagged Robo1 rescue transgene stained with anti-HA and anti-HRP. The HA expression pattern closely resembles that of endogenous Robo1. (B) Stage 17 embryo expressing the genomic HA-tagged Robo1 $\Delta$ WIRS rescue transgene showing comparable HA staining. 


\section{FIGURE S5}

A

EXON 16

INTRON 16

EXON 17

robo cgtaaccttaccaccttctacaattgtcgcaaggtgaggatcatatgaattgcatcacacaacaatttcttataggaaatattctttcagagccccgataatcccacg

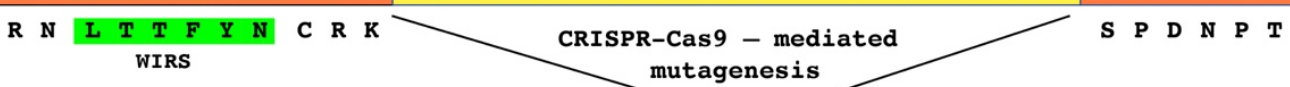

robo $\triangle W I R S$

gtaacgctaccgcettcgct---tgtcgcaagagcccogataatcccacg

$\mathbf{R}$ N
WIRS
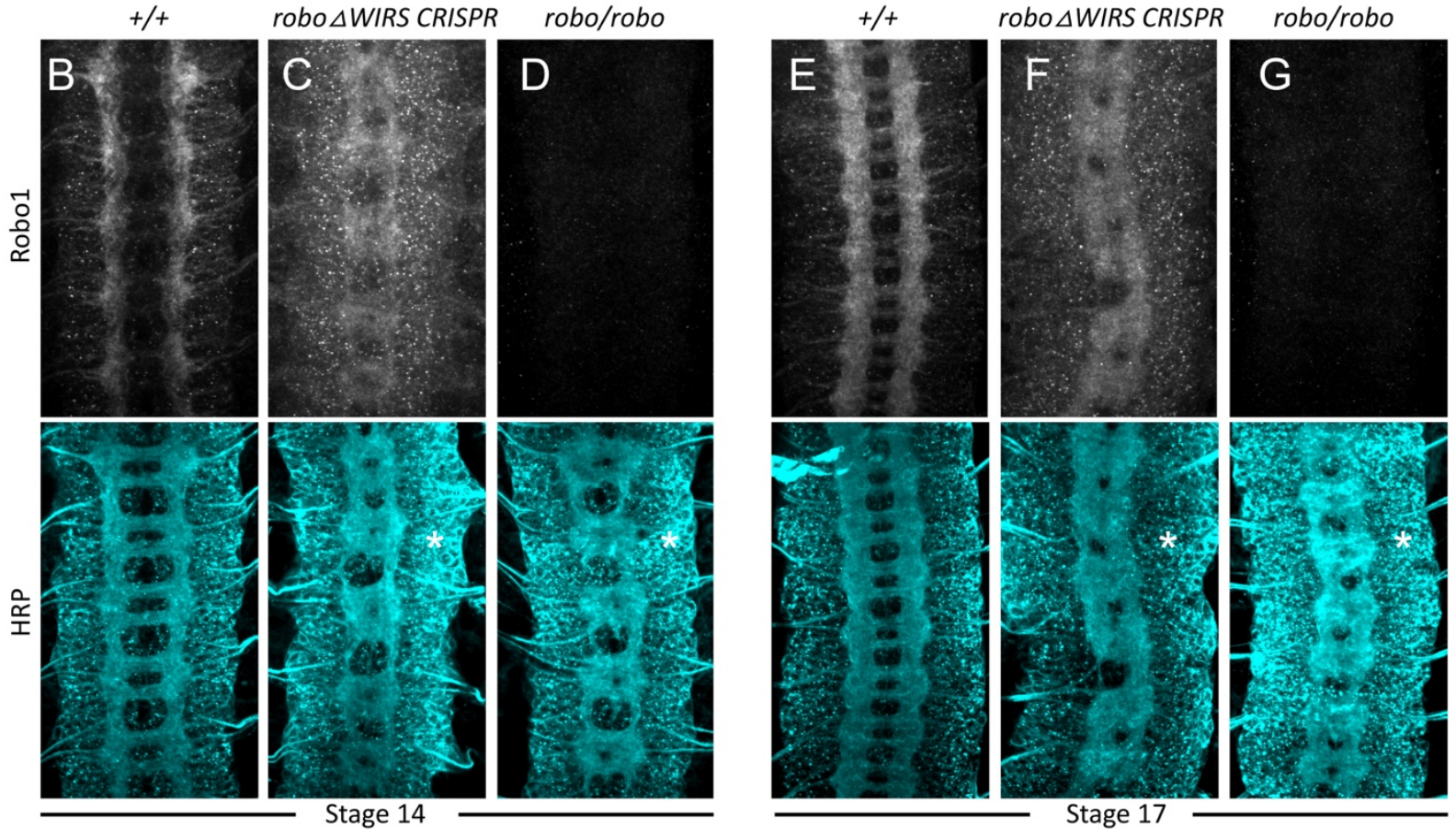

Supplementary figure 5. Schematic for CRISPR-Cas9 mutagenesis and Robo1 staining in CRIPR robo $\Delta$ WIRS embryos. (A) Schematic for CRSIPR-Cas9 mutagenesis of the WIRS motif in the endogenous robo1 locus. Four amino acids in the WIRS motif were mutated and deletion of the small intron 16 occurred during mutagenesis. (B and C) Stage 17 embryos stained with antiRobo1 and anti-HRP. (B) Wild type embryos show Robo1 staining enriched in the longitudinal tracts and no HRP phenotype. (C) CRISPR robo $\triangle$ WIRS embryos show decreased Robo1 staining compared to wild type however Robo1 can still be detected on axons in these embryos. There is a strong loss-of-repulsion phenotype in HRP with thickening and fusion of commissures (asterisk). 


\section{FIGURE S6}

hRobo1-MYC + Slit
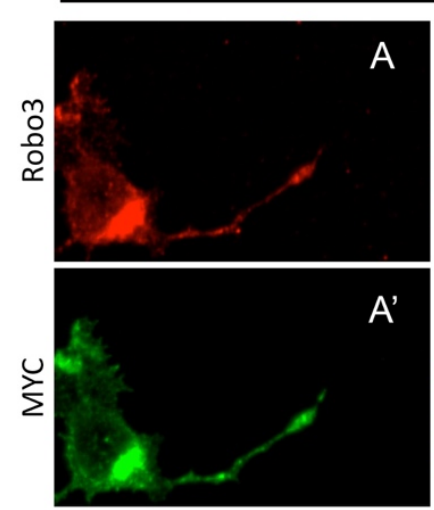
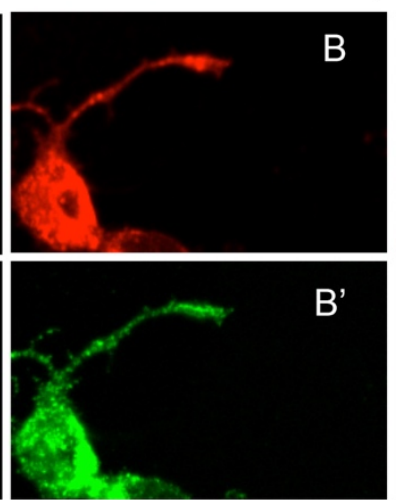

hRobo1WIRS-MYC + Slit
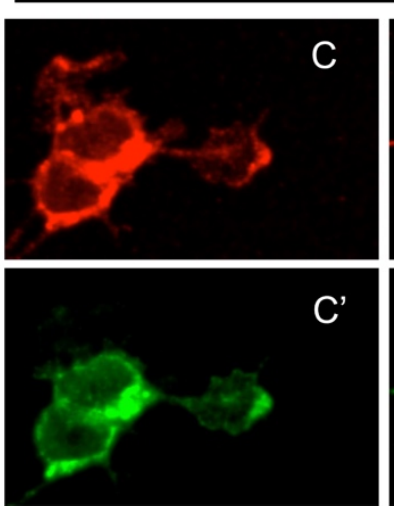

Supplementary figure 6. Expression of hRobo1 variants electroporated into dorsal spinal commissural neurons. (A-D) Slit-treated E12 dissociated mouse dorsal commissural neurons stained with anti-Robo3 and ( $\mathbf{A}^{\prime}$ - $\mathbf{D}^{\prime}$ ) anti-MYC to label commissural neurons electroporated with MYC-tagged hRobo1 variants. Wild type hRobo1 and hRobo1 $\Delta$ WIRS show comparable levels of MYC staining. 